\title{
Solution Structures of Conformationally Equilibrium Forms of Holo-Acyl Carrier Protein (PfACP) from Plasmodium falciparum Provides Insight into the Mechanism of Activation of ACPs ${ }^{\dagger, *}$
}

\author{
Alok Kumar Sharma, ${ }^{\S}$ Shailendra Kumar Sharma, ${ }^{\S}$ Avadhesha Surolia, ${ }^{\S}$ Namita Surolia," and Siddhartha P. Sarma*,§ \\ Molecular Biophysics Unit, Indian Institute of Science, Bangalore 560012, India, and Molecular Biology and Genetics Unit, \\ Jawaharlal Nehru Centre for Advanced Scientific Research, Jakkur, Bangalore 560064, India
}

Received February 22, 2006; Revised Manuscript Received April 2, 2006

\begin{abstract}
Acyl Carrier Protein (ACP) from the malaria parasite, Plasmodium falciparum (PfACP) in its holo form is found to exist in two conformational states in solution. Unique 3D solution structures of holo-PfACP have been determined for both equilibrium conformations, using high-resolution NMR methods. Twenty high-resolution solution structures for each of the two forms of holo-PfACP have been determined on the basis of 1226 and 1218 unambiguously assigned NOEs (including NOEs between $4^{\prime}$-phosphopantetheine prosthetic group (4'-PP) and protein), 55 backbone dihedral angles and 26 hydrogen bonds. The atomic rmsd values of the determined structures of two equilibrium forms, about the mean coordinates of the backbone and heavy atoms, are $0.48 \pm 0.09$ and $0.92 \pm 0.10$ and $0.49 \pm 0.08$ and $0.97 \pm 0.11 \AA$, respectively. The interaction of 4'-PP with the polypeptide backbone is reported here for the first time for any of the ACPs. The structures of holo-PfACP consist of three well-defined helices that are tightly packed. The structured regions of the molecule are stabilized by extensive hydrophobic interactions. The difference between the two forms arises from a reorientation of the 4'-PP group. The enthalpy difference between the two forms, although small, implies that a conformational switch is essential for the activation of holo-ACP. Sequence and structures of holo-PfACP have been compared with those of the ACPs from type I and type II fatty acid biosynthesis pathways (FAS), in particular with the ACP from rat and the butyryl-ACP from E. coli. The PfACP structure, thus determined has several novel features hitherto not seen in other ACPs.
\end{abstract}

Plasmodium falciparum is responsible for the most virulent form of malarial infection (1). Current measures for the treatment of malarial infections is compounded by the ability of this organism to develop resistance to known antimalarial drugs $(2,3)$. The need for new and novel targets for drugs for treating malaria therefore need no emphasis. One class of molecules that could prove to be valuable targets for the design of antimalarial drugs are the enzymes of the fatty acid biosynthetic pathway $(\mathrm{FAS})^{1}(4,5)$. The role of fatty acids for the normal physiology of living organisms is well documented (6). Two types of pathways are known to exist in living organisms. The FAS I (type I or associative)

\footnotetext{
$\doteqdot$ This work was supported by a grant from the Department of Biotechnology (DBT), Government of India to N.S.

$\$$ The atomic coordinates for the ensemble of 20 structures and distance and dihedral angle constraints of major and minor conformations of holo-PfACP have been deposited in the Protein Data Bank (http://www.rcsb.org.) under pdb codes $2 \mathrm{FQ} 0$ and $2 \mathrm{FQ} 2$. The ${ }^{1} \mathrm{H},{ }^{15} \mathrm{~N}$, and ${ }^{13} \mathrm{C}$ chemical shift values of major and minor conformations of holo-PfACP have been deposited in BioMagResBank (http://www. bmrb.wisc.edu) under accession number 6516.

* To whom correspondence should be addressed. Tel: 91-8022932839. Fax: 91-80-23600535. E-mail: sidd@mbu.iisc.ernet.in.

$\S$ Indian Institute of Science.

"Jawaharlal Nehru Centre for Advanced Scientific Research.

${ }^{1}$ Abbreviations: FAS, fatty acid biosynthesis; ACP, acyl carrier protein; holo-PfACP, holo-acyl carrier protein from Plasmodium falciparum; 4'-PP, 4'-phosphopantetheine; DTT, dithiothreitol; DSS, sodium 4,4-dimethyl-4-silapentanesulfonate; CSI, chemical shift index; NOESY-HSQC, nuclear overhauser effect spectroscopy-heteronuclear single quantum coherence; RMSD, root-mean-square deviation.
}

pathway is found in mammals, fungi, and some mycobacteria, whereas the FAS II (type II or dissociative) pathway is found in bacteria and plants. A point of interest is that the host and the malaria parasite each have distinct fatty acid biosynthetic pathways, that is, the type I pathway is present in the host, whereas the type II pathway is present in the parasite. Given the difference between the two FAS pathways, the type II pathway has emerged as a potential target for the development of novel antimalarial agents (7).

A critical protein for fatty acid biosynthesis is the acyl carrier protein (ACP) (8). ACPs, are small, acidic, and conserved proteins, which in their holo form serve to shuttle the lengthening fatty acid substrates to the various catalytic sites of the enzymes involved in type II FAS. The growing acyl chains are covalently attached to the terminal sulfhydryl group of the 4'-phosphopantetheine (4'-PP) arm of holo-ACP. Type II FAS ACPs from several sources, for example, Escherichia coli ACP (9-12), Bacillus subtilis ACP (13), and M. tuberculosis ACP (14), have been characterized, and the solution structures have been determined. Only one structure is known of an ACP from the FAS I pathway, that of the rat FAS ACP (15). Structures of ACPs that are involved in polyketide synthesis (PKS), such as those of Streptomyces coelicolor actinorhodin apo-ACP (16), frenolicin ACP from Streptomyces roseofulvus (17), and oxytetracycline ACP from Streptomyces rimosus (18), have also been determind by NMR methods. The structures of $E$. coli apo-ACP (19), E. coli butyryl-ACP (20), and the ACP- 
ACPS complex from $B$. subtilis (21) have been determined at high resolution by X-ray diffraction methods. Structural studies of ACPs have shown that these proteins are characterized by a four helix bundle in which helix II plays the major role in $\mathrm{ACP}$ - protein interactions. The majority of the residues in helix II are conserved or similar in all known ACPs. An important feature of the residues in helix II is the conserved DSL motif close to its $N$-terminus, in which the central serine serves to attach the 4'-PP arm (22). Despite considerable similarity in their amino acid sequences, secondary structural elements, and overall topologies, certain ACPs are known to exist in solution in conformational equilibrium in two isomeric forms that are in slow exchange on the NMR time scale $(17,23-24)$. On the basis of this conformational equilibria, Prestegard and co-workers have proposed a dynamic model for the structure of E. coli ACP (23).

We report here the high-resolution solution structures of holo-ACP from P. falciparum (PfACP) obtained using highresolution multidimensional and multinuclear NMR methods. The structures of holo-PfACP are unique in that NMR derived experimental restraints were obtained on holo-protein samples containing a uniformly ${ }^{13} \mathrm{C} /{ }^{15} \mathrm{~N}$-enriched $4^{\prime}-\mathrm{PP}$ prosthetic group that was biosynthetically obtained. HoloPfACP is found to exist in two conformational states in solution that are in slow exchange on the NMR time scale. We also show that the two states arise from differences in prosthetic group orientations, which manifest as chemical shift heterogeneity in the various NMR spectra. The determination of the structures of this protein assumes significance because it enables the comparison of the two forms of the protein with structures of other ACPs that participate in type I and type II FAS pathways. Knowledge of the 3D structure of PfACP will help in probing the details of the interaction between ACP and other enzymes of type II FAS, thereby opening possibilities for the design of molecules that disrupt these interactions, which would in turn prevent the growth of the parasite.

\section{MATERIALS AND METHODS}

Cloning and Overexpression. The cloning, expression, and purification of holo-PfACP in E. coli has been presented elsewhere (25). The construct used for the NMR structural studies contains four more residues at the $N$-terminus that have arisen out of cloning exigencies. Uniformally ${ }^{13} \mathrm{C} /{ }^{15} \mathrm{~N}$ and ${ }^{15} \mathrm{~N}$-enriched protein samples were prepared by growing the cells in M9 minimal media (26) containing ${ }^{15} \mathrm{NH}_{4} \mathrm{Cl}(1$ $\mathrm{g} / \mathrm{L}) /{ }^{13} \mathrm{C}_{6}$-glucose $(2 \mathrm{~g} / \mathrm{L})$ or ${ }^{15} \mathrm{NH}_{4} \mathrm{Cl}$ (Cambridge Isotopes Labs, U.S.A.) as the sole source of nitrogen and carbon, respectively. The $\mathrm{N}$-terminal $\mathrm{His}_{6}$-tag was removed by incubating the holo-protein with thrombin $(1 \mathrm{U} / \mathrm{mg})$ for $2 \mathrm{~h}$ at $22{ }^{\circ} \mathrm{C}$. The cleaved protein was further purified by metal affinity chromatography. The Plasmodium protein thus overexpressed and purified was present in its $100 \%$ holo form. The isotopic enrichment of the parasite holo-ACP is unique in that the biosynthetically driven $4^{\prime}$-PP moiety is also isotopically enriched. The purity and homogeneity of the holo-PfACP sample was verified by SDS-PAGE and by electrospray mass spectrometry. Mass spectrometric analysis showed the holo-ACP to be $98 \%$ enriched in carbon13 and nitrogen- 15 .
NMR Spectroscopy. The isotopically enriched samples for NMR spectroscopy were prepared by dialyzing the purified holo-protein into a $50 \mathrm{mM}$ sodium phosphate buffer at $\mathrm{pH}$ 6.5 containing $100 \mathrm{mM} \mathrm{NaCl}, 0.5 \% \mathrm{NaN}_{3}$, and $2 \mathrm{mM}$ DTT. The dialyzed protein was then concentrated using Centricon (5.0 kDa NMWCO). Samples for NMR were 1.2-1.4 mM in concentration in a $90 \% \mathrm{H}_{2} \mathrm{O} / 10 \% \mathrm{D}_{2} \mathrm{O}$ mixture or in $99 \%$ $\mathrm{D}_{2} \mathrm{O}$. One mM DSS was added as an internal chemical shift reference standard.

All NMR experiments were performed either on a Varian INOVA $500 \mathrm{MHz}$ spectrometer equipped with a triple resonance ( $z$-axis) pulsed field gradient probe or on Bruker Avance $700 \mathrm{MHz}$ spectrometer equipped with a $5 \mathrm{~mm}$ cryogenically cooled triple resonance PFG ( $z$-axis) probe. The sample temperature was maintained at $300 \mathrm{~K}$ during all experiments. All of the NMR spectra were acquired in the gradient-selected sensitivity enhanced mode. Frequency discrimination in the indirect dimensions of all multidimensional spectra was achieved using the States-TPPI (27) mode of quadrature detection.

NMR data were processed on an Intel PC workstation running Suse Linux 8.2 using NMRPipe/NMRDraw processing software (28). The directly and indirectly detected time domain data of $2 \mathrm{D}$ and 3D spectra were processed by applying a $90^{\circ}$ phase-shifted squared sinebell or a Gaussian filter with a line-broadening parameter of $10 \mathrm{~Hz}$ as weighting functions. Data sets were zero filled once in each dimension prior to Fourier transformation. All chemical shifts were referenced to the DSS internal standard. The NMR spectra were visualized and analyzed using $\operatorname{ANSIG}(29,30)$.

The resonance assignments for the protein backbone, its side chain, and all $\mathrm{H}, \mathrm{C}$, and $\mathrm{N}$ resonances of the ${ }^{13} \mathrm{C} /{ }^{15} \mathrm{~N}-$ enriched 4'-PP prosthetic group attached to serine-37 of the protein were unambiguously assigned using the data from 2D spectra, viz., ${ }^{1} \mathrm{H}-{ }^{15} \mathrm{~N}$ HSQC, ${ }^{1} \mathrm{H}-{ }^{13} \mathrm{C}$ CT-HSQC, $\left[{ }^{1} \mathrm{H},{ }^{1} \mathrm{H}\right]$ $\operatorname{TOCSY}\left(\tau_{\mathrm{m}}=52.5 \mathrm{~ms}\right)$, and $\left[{ }^{1} \mathrm{H},{ }^{1} \mathrm{H}\right] \operatorname{NOESY}\left(\tau_{\mathrm{m}}=75\right.$ and $125 \mathrm{~ms}$ ); triple resonance correlation spectra, viz., HNCACB, $\mathrm{CBCA}(\mathrm{CO}) \mathrm{NH}, \mathrm{HNCO}, \mathrm{CC}(\mathrm{CO}) \mathrm{NH}-\mathrm{TOCSY}$, and $\mathrm{H}(\mathrm{C}-$ CCO)NH-TOCSY; and 3D spectra of ${ }^{15} \mathrm{~N}$-edited NOESY$\operatorname{HSQC}\left(\tau_{\mathrm{m}}=120 \mathrm{~ms}\right)$ and ${ }^{13} \mathrm{C}$-edited NOESY-HSQC $\left(\tau_{\mathrm{m}}=\right.$ $120 \mathrm{~ms})(31-33)$. These assignments were corroborated with assignments from double-resonance experiments, such as HCCH-TOCSY $\left(\tau_{\mathrm{m}}=17 \mathrm{~ms}\right)(31),{ }^{15} \mathrm{~N}$-edited TOCSYHSQC $\left(\tau_{\mathrm{m}}=50 \mathrm{~ms}\right)(31)$. The ring protons of aromatic residues were assigned using a combination of 2D TOCSY and 2D NOESY spectra on a sample of holo-PfACP in $99 \%$ $\mathrm{D}_{2} \mathrm{O}$. Scalar coupling constants, ${ }^{3} \mathrm{~J}_{\mathrm{H}} \mathrm{N}_{\mathrm{H}}^{\alpha}$ were calculated from measured diagonal and cross-peak intensities in a 3D HNHA spectrum (34).

The difference in the free enthalpies for the two equilibrium forms were estimated using the following equation (35) where $I_{\mathrm{A}}$ and $I_{\mathrm{B}}$ are the cross-peak intensities of the isomeric

$$
\Delta G=-R T \ln K ; K=\frac{\left[I_{\mathrm{A}}\right]}{\left[I_{\mathrm{B}}\right]}
$$

forms in the ${ }^{1} \mathrm{H}^{15} \mathrm{~N}$ HSQC spectrum.

${ }^{15} \mathrm{~N}$ Backbone Dynamics. The 2D ${ }^{1} \mathrm{H}^{-15} \mathrm{~N}$ HSQC spectra to measure relaxation rates $T_{1}, T_{2}$, and heteronuclear $\left\{{ }^{1} \mathrm{H}\right\}$ ${ }^{15} \mathrm{~N}$ NOE were recorded at $500 \mathrm{MHz}$ on a ${ }^{15} \mathrm{~N}$-labeled sample using the pulse programs described by Farrow et al. (36). The $T_{1}$ data were acquired with $5 \mathrm{~s}$ recycle delays and 
relaxation delays of $10,50,100,200,500,800,1000,1200$, and $1500 \mathrm{~ms} . T_{2}$ data were acquired with $5 \mathrm{~s}$ recycle delays and relaxation delays of $15.5,31,46.562,93,108.5,139.5$, $155.0,170.5$, and $186 \mathrm{~ms} . T_{1}$ and $T_{2}$ values were determined by fitting peak heights using the nonlinear least-squares (NlinLS) routine that is part of the NMRPipe/NMRDraw distribution. $\left\{{ }^{1} \mathrm{H}\right\}-{ }^{15} \mathrm{~N}$ NOEs were determined as the ratio of peak heights in the spectrum recorded with ${ }^{1} \mathrm{H}$ saturation (NOE) (using a $3 \mathrm{~s}$ recycle delay and $2 \mathrm{~s}$ proton saturation) to that of the spectrum recorded without ${ }^{1} \mathrm{H}$ saturation (NONOE) (using a $5 \mathrm{~s}$ recycle delay). $T_{1}, T_{2}$, and heteronuclear NOE data were analyzed using the Lipari-Szabo model free formalism $(37,38)$ with the program Modelfree v4.01 $(39,40)$.

Constraints Generation and Structure Determination of Conformational Isomers of Holo-PfACP. Correlation peaks that were unambiguously and uniquely assigned to the two conformational forms in 2D $\left[{ }^{1} \mathrm{H},{ }^{1} \mathrm{H}\right]$ NOESY, 3D- ${ }^{15} \mathrm{~N}$ resolved $\left[{ }^{1} \mathrm{H},{ }^{1} \mathrm{H}\right]$ NOESY, and ${ }^{13} \mathrm{C}$-resolved $\left[{ }^{1} \mathrm{H},{ }^{1} \mathrm{H}\right]$ NOESY spectra were integrated, and on the basis of the integrated intensities, NOEs were classified as strong, medium, weak, and very weak with upper bounds of 3.5, 4.0, 5.0, and $6.0 \AA$ respectively. All distance restraints employed a lower bound of $1.80 \AA$. Hydrogen-bond restraints were generated for regions that were defined to be $\alpha$-helical in secondary structure, on the basis of medium-range NOEs. The upper distance bound employed for the hydrogen bonds were set to $2.2 \AA$ for $\mathrm{HN}-\mathrm{O}$ and $3.2 \AA$ for $\mathrm{N}-\mathrm{O}$.

Backbone dihedral angles $(\phi, \psi)$ were estimated by use of the CaAngleRestraints.cya routine in CYANA-2.1 (41) and the program TALOS $(42)$ in which ${ }^{13} \mathrm{C}^{\alpha}$-chemical shifts and the ${ }^{1} \mathrm{H}^{\alpha}-,{ }^{13} \mathrm{C}^{\alpha}-,{ }^{13} \mathrm{C}^{\beta}-,{ }^{13} \mathrm{C}^{\prime}$-, and ${ }^{15} \mathrm{~N}$-chemical shifts, respectively, were used as input for the predictions by the two programs. The predicted backbone dihedral angles obtained from the two programs were in good agreement, and hence, the output from the CaAngleRestraints.cya routine was used as input for structure calculations. Because the ${ }^{13} \mathrm{C}$-chemical shifts were found to be identical in the case of residues that exhibited the backbone amide proton-amide nitrogen chemical shift heterogeneity, the protein backbone dihedral angles were assumed to be the same for the two forms.

Three-dimensional structures were calculated with the program CYANA-2.1 on the basis of experimentally derived distances from NOEs, hydrogen-bond distances and dihedralangle restraints, using the torsion-angle dynamics protocol. The final NOE data sets reflected all of the assigned NOEs for both forms of holo-PfACP. In the process of structure determination, 200 random conformers were subjected to 12000 steps of annealing to obtain an ensemble of 20 of the best structures of acceptable stereochemical quality for each equilibrium form.

Structure Evaluation. The stereochemical qualities of the calculated structures were assessed using CYANA and PROCHECK-NMR (43) and compared with those of ACPs belonging to the FAS and PKS families, using MOLMOL (44), VMD (45), and GRASP (46). The protein sequences were aligned in ClustalW. The percentage of sequence identities were determined from the LALIGN programe (47).

\section{RESULTS}

Sequence Specific Assignments. The holo-ACP from the malaria parasite $P$. falciparum (PfACP) exists in two conformational states in solution that are in slow exchange on the NMR time scale. The ${ }^{1} \mathrm{H}-{ }^{15} \mathrm{~N}$ 2D HSQC spectrum of holo-PfACP (Figure 1) exhibits well-dispersed resonances for nearly all backbone and side-chain proton-nitrogen pairs. Backbone amide resonances of residues D36 and I55 were found to be extremely weak in the ${ }^{1} \mathrm{H}^{-15} \mathrm{~N}$ HSQC, presumably because of the exchange with the bulk solvent. These resonances were, however, easily assigned through the tripleresonance spectra and the $3 \mathrm{D}{ }^{1} \mathrm{H}-{ }^{15} \mathrm{~N}$ NOESY-HSQC. Also indicated in the figure are the assignments for 11 pairs of side-chain amide cross-peaks arising from 6 aspargines and 5 glutamines. The cross-peaks of the backbone and the 4'PP proton-nitrogen pairs that arise because of the conformational exchange are indicated in Figure 1. The identity and origin of these cross-peaks will be discussed below.

Resonance Assignment of the 4'-PP Prosthetic Group. Unambiguous assignments for ${ }^{1} \mathrm{H},{ }^{15} \mathrm{~N}$, and ${ }^{13} \mathrm{C}$ nuclei in $4^{\prime}-$ PP are reported in Table 1 . The ${ }^{1} \mathrm{H}-,{ }^{15} \mathrm{~N}$-, and ${ }^{13} \mathrm{C}$-chemical shifts and ${ }^{3} J_{\mathrm{H}}{ }^{\mathrm{N}}{ }_{\mathrm{H}}^{\alpha}$ scalar coupling constants, of holo-PfACP have been reported previously (BMRB accession number 6516) (48).

Conformational Exchange. During the course of sequential assignments, it was found that residues V41, A60, L61, and the 4'-PP moiety exhibited conformational exchange between two states, the rate of exchange being slow on the NMR time scale. In the strip plots of the ${ }^{15} \mathrm{~N}$ NOESY-HSQC spectrum (Figure 2), two sets of resonances can be clearly observed for V41, A60, L61, and 4'-PP. The chemical shifts of the two forms of these residues are fully resolved in the HN chemical shift, are identical in the carbon chemical shift, and nearly identical in the nitrogen chemical shift. The assignment of these resonances to conformationally exchanged residues was confirmed through an analysis of $\mathrm{HNCACB}, \mathrm{CBCA}(\mathrm{CO}) \mathrm{NH}$, and $\mathrm{CC}(\mathrm{CO}) \mathrm{NH}$. An integration of HSQC cross-peaks for the two equilibrium forms showed that the two forms exist as an equilibrium mixture in an approximately 65:35 ratio. The identified major (A) and minor (B) forms for V41, A60, L61, and the amide protons of $4^{\prime}-\mathrm{PP}\left(4^{\prime}-\mathrm{PP}^{\mathrm{c}}\right.$ and $4^{\prime}-\mathrm{PP}^{\mathrm{g}}$ (cf. Table 1)) are assigned in the ${ }^{1} \mathrm{H}-{ }^{15} \mathrm{~N}$ correlation spectrum (Figure 1). The amide proton resonances of these residues are separated by $0.06(42 \mathrm{~Hz})$, $0.04(28 \mathrm{~Hz}), 0.06(39 \mathrm{~Hz}), 0.06(45 \mathrm{~Hz})$, and $0.05 \mathrm{ppm}(34$ $\mathrm{Hz}$ ), respectively, in the ${ }^{1} \mathrm{H}-{ }^{15} \mathrm{~N}$ HSQC spectrum (digital resolution of $5.5 \mathrm{~Hz} / \mathrm{pt}$ in $\mathrm{F} 2$ ). In contrast, the amide nitrogen resonances are separated by $0.0,0.17(12 \mathrm{~Hz}), 0.0,0.0$, and $0.26 \mathrm{ppm}(18.2 \mathrm{~Hz})$ (digital resolution of $9.7 \mathrm{~Hz} / \mathrm{pt}$ in F1). However, the $\mathrm{H}^{\alpha}$ and $\mathrm{C}^{\alpha}$ resonances of V41, A60, and L61 exhibit identical chemical shifts in the ${ }^{15} \mathrm{~N}$-edited TOCSYHSQC and ${ }^{1} \mathrm{H}-{ }^{13} \mathrm{C}$ HSQC spectra, respectively. The difference in free enthalpies for the two equilibrium forms (A and B) was found to be $\sim 0.5 \mathrm{kcal} / \mathrm{mol}$.

4'-PP Moiety and Its Interaction with Protein. The prosthetic group 4'-PP attached to S37 spatially interacts with the S37, L38, and D39 residues of the polypeptide, and these interactions have been identified for both the A and B forms of 4'-PP. The NOESY strip plots of Figure 3 show the NOE interaction between the various nuclei of $4^{\prime}$-PP and the different protons of the S37, L38, and D39 residues. The minor form of V41 interacts with $4^{\prime}$-PP resulting in an additional restraint for the minor conformation structure determination (Figure 2). Of residues V41, A60, and L61, only V41 shows NOE contacts with the prosthetic group. 


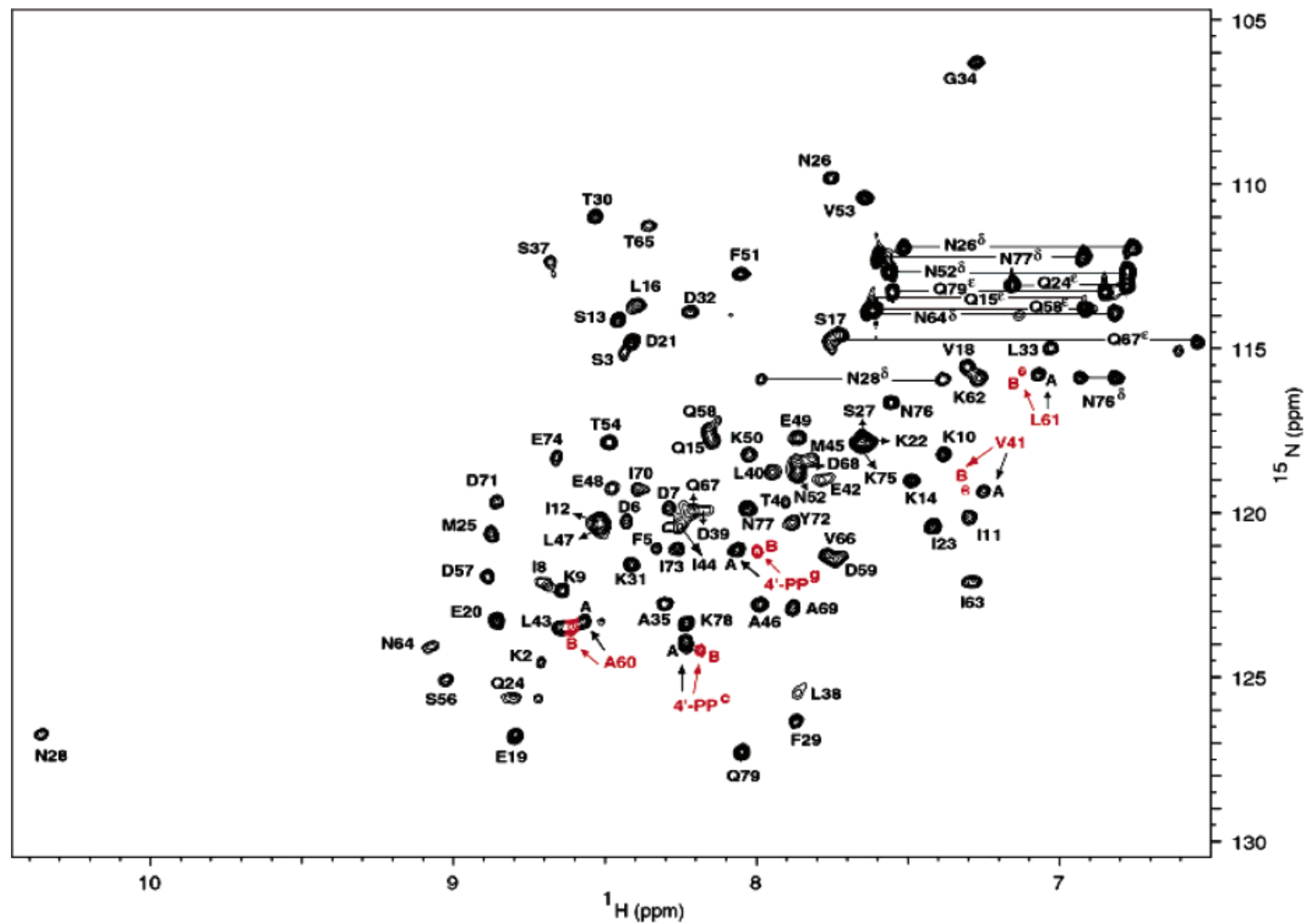

FIGURE 1: Two-dimensional ${ }^{1} \mathrm{H}_{-}{ }^{15} \mathrm{~N}$ HSQC spectrum of holo-PfACP showing correlations between backbone and side chain protonnitrogen pairs. The cross-peaks have been labeled using a one letter amino acid code in a sequence specific manner. Proton-nitrogen pairs from the side chains of Gln and Asn are connected by horizontal lines. Also indicated in the spectrum are the major (A) and minor (B) forms of residues V41, A60, and L61. The major and minor forms of the proton-nitrogen correlations arising from $\beta$-mercapto-ethylamine $\left(4^{\prime}-\mathrm{PP}^{\mathrm{c}} \mathrm{A}, \mathrm{B}\right)$ and $\beta$-alanine (4'-PPg $\left.\mathrm{A}, \mathrm{B}\right)$ moieties of the $4^{\prime}$-phosphopantetheine group are labeled accordingly (cf. Table 1$)$. For clarity, the minor forms (B) are shown and labeled in red.

Table 1: Chemical Shifts of NMR Assignable Heavy Atoms of 4'-Phosphopantetheine $\left(4^{\prime}-\mathrm{PP}\right)^{a}$

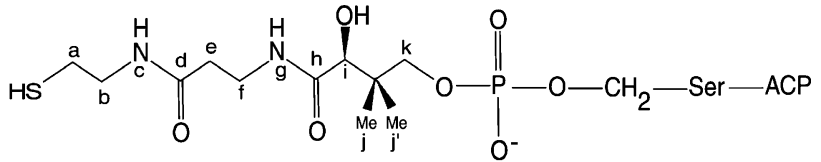

$\mathrm{Me}=$ methyl group

\begin{tabular}{|c|c|c|c|}
\hline position & ${ }^{1} \mathrm{H}(\mathrm{ppm})$ & ${ }^{13} \mathrm{C}$ (ppm) & ${ }^{15} \mathrm{~N}(\mathrm{ppm})$ \\
\hline$a^{b}$ & 2.79 & 39.47 & \\
\hline $\mathrm{b}^{b}$ & 3.46 & 38.08 & \\
\hline $\mathrm{c}$ & $8.23(8.18)^{c}$ & & $123.93(124.19)^{\circ}$ \\
\hline $\mathrm{d}$ & & 176.50 & \\
\hline $\mathrm{e}^{b}$ & 2.48 & 41.18 & \\
\hline $\mathrm{f}^{b}$ & 3.49 & 38.17 & \\
\hline g & $8.06(7.99)^{c}$ & & $121.12(121.16)^{c}$ \\
\hline $\mathrm{h}$ & & 177.57 & \\
\hline $\mathrm{i}$ & 4.00 & 76.78 & \\
\hline $\mathrm{j}, \mathrm{j}^{\prime}$ & $0.84,0.93$ & $20.53,22.55$ & \\
\hline $\mathrm{k}$ & $3.79,3.38$ & 74.24 & \\
\hline
\end{tabular}

${ }^{a}$ Atom wise ${ }^{1} \mathrm{H},{ }^{13} \mathrm{C}$, and ${ }^{15} \mathrm{~N}$ assignments are shown as labeled in the structure of $4^{\prime}-\mathrm{PP}$, shown above the Table. All assignments were made using the triple-resonance data sets described in the text).

${ }^{b}$ Methylene protons show chemical shift degeneracy. ${ }^{c}$ Values shown in parentheses are for the minor conformation of holo-PfACP.

None of the other residues that exhibit heterogeneity show NOEs to $4^{\prime}$-PP. In all, five and six unambiguous NOE contacts between $4^{\prime}$-PP and the protein and five and six intra $4^{\prime}$-PP NOEs could be assigned for the major and minor conformations, respectively. These NOE distance restraints, from and within $4^{\prime}$-PP, were critical in defining the orientation of the prosthetic group in the major and minor conformations of holo-PfACP, respectively. A large number of NOEs defining the two forms could be easily assigned from the ${ }^{15} \mathrm{~N}$-edited NOESY spectrum because of wellresolved conformationally exchanged cross-peaks in the ${ }^{1} \mathrm{H}$ ${ }^{15} \mathrm{~N}$ HSQC spectrum (vide supra). Degeneracy in aliphatic ${ }^{1} \mathrm{H}$ - and ${ }^{13} \mathrm{C}$-chemical shifts precludes the assignment of unique NOEs to the conformationally exchanged forms in the ${ }^{13} \mathrm{C}$-edited NOESY spectrum.

Secondary Structures of Major and Minor Conformations of Holo-PfACP. The solution secondary structures of major and minor forms of holo-PfACP were deduced from computed ${ }^{1} \mathrm{H}^{\alpha}-,{ }^{13} \mathrm{C}^{\alpha}-,{ }^{13} \mathrm{C}^{\beta}$ - and ${ }^{13} \mathrm{C}^{\prime}$-secondary shifts using the consensus chemical shift index (CSI) method (49). The major and minor conformations adopt three well-defined major helical regions (HI, HII, and HIII) and one small helix (HII') (Figure 4). Identification of medium-range NOEs and estimated ${ }^{3} J_{\mathrm{H}} \mathrm{N}_{\mathrm{H}}^{\alpha}$ scalar coupling constants provided corroborative evidence for the helices detected by CSI. Figure 4 summarizes short- and medium-range NOE patterns obtained for both conformations of holo-PfACP. Almost identical $\mathrm{C}^{\alpha}$ - and $\mathrm{C}^{\beta}$-chemical shifts indicate that the secondary structural elements are largely unaffected by conformational isomerism.

Distance and Dihedral Restraints. NMR-derived experimental restraints for the determination of the tertiary 

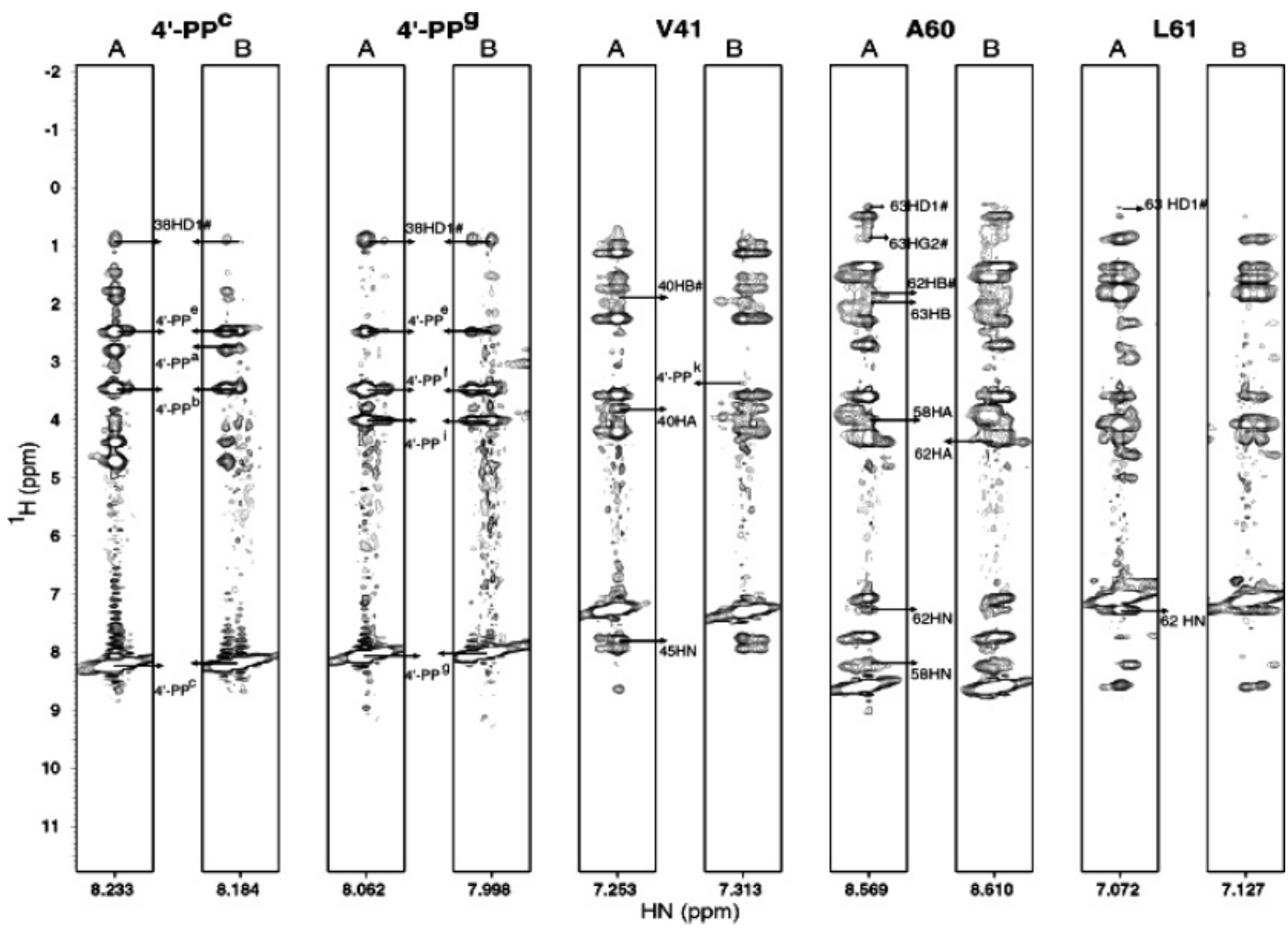

FIGURE 2: Strip plots from the 3D ${ }^{15} \mathrm{~N}$-edited NOESY-HSQC spectrum showing NOEs to the conformationally exchanged forms (labeled as A and B) of residues V41, A60, L61, and both amides of the $4^{\prime}-\mathrm{PP}\left(4^{\prime}-\mathrm{PP}^{\mathrm{c}}\right.$ and $\left.4^{\prime}-\mathrm{PP}^{\mathrm{g}}\right)$ of holo-PfACP. The strips are centered at the amide-proton chemical shift of each residue at the corresponding nitrogen chemical shift. The NOEs unique to each form of the protein residues are indicated, whereas all assigned NOEs to the prosthetic group are labeled.

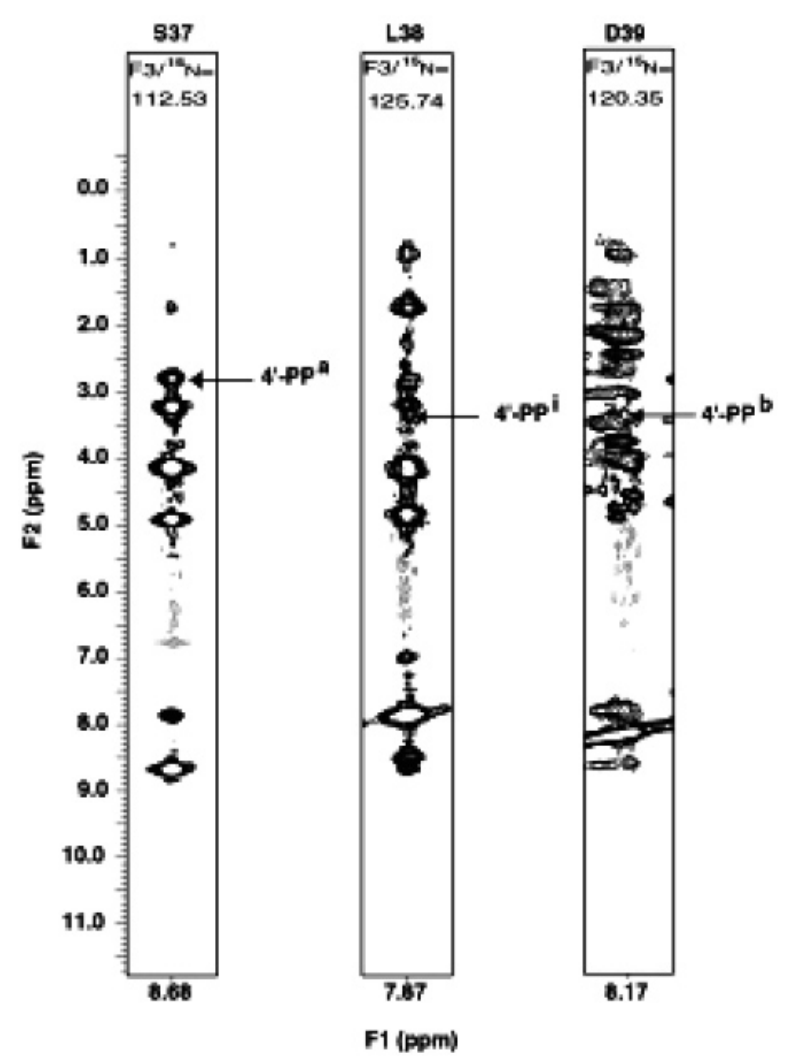

FIGURE 3: Strip plots from the 3D ${ }^{15} \mathrm{~N}$-edited NOESY-HSQC spectrum showing NOE correlations between residues S37, L38, and D39 and $4^{\prime}-\mathrm{PP}$. The strips are plotted at respective $\mathrm{H}^{\mathrm{N}_{-}}$and ${ }^{15} \mathrm{~N}$-chemical shifts.

structures of major and minor conformations of holo-PfACP consisted of 1226 and 1218 unique and nontrivial NOEs (including NOE distances due to the $4^{\prime}$-PP group) and 55 $(\phi, \psi)$-backbone angle restraints along with 26 hydrogen-bond restraints, which were generated for the residues in the helical regions. A total of 146 long-range NOEs were assigned for both major and minor conformations, out of which 74 are from $\left(\mathrm{H}^{\alpha}\right.$ and $\left.\mathrm{H}^{\mathrm{N}}\right)$ backbone-side chain interactions, 61 are from side chain-side chain interactions, and 11 are from backbone-backbone interactions. Aromatic residues F5 (helix HI), F29 (loop LI), F51 (helix HII), and Y72 (helix HIII) contributed to 55 long-range contacts, out of which, 41 served to constrain the backbone of the helical residues, and the remaining ones were involved in constraining the corresponding loop regions. Three bond ${ }^{3} J_{\mathrm{H}} \mathrm{N}_{\mathrm{H}}{ }^{\alpha}$ coupling constants and almost identical ${ }^{13} \mathrm{C}^{\alpha}$-chemical shifts indicated that the backbone torsional angles for the major and minor forms of the conformationally exchanged residues were the same.

Three-Dimensional Structure of Holo-PfACP. Figure 5(A and $C$ ) shows the superposition of 20 of the best conformers of each major and minor conformations of holo-PfACP when superimposed on their backbone $\left(\mathrm{N}, \mathrm{C}^{\alpha}\right.$, and $\left.\mathrm{C}^{\prime}\right)$ atoms. The criterion for the selection of the 20 best structures was based on these structures having the lowest target functions, no upper distance NOE violations greater than $0.5 \AA$, and no dihedral angle violations greater than $5^{\circ}$. This is a clear indication of the good correlation between NMR experimental data and calculated structures. The representative structures of the major and minor conformations of holoPfACP are shown in ribbon representation in Figure 5 (B and D). The mean solution structure of each conformer is composed of three well-defined helices HI (T4 -Q15), HII (S37-F51), and HIII (V66-K75) and one short helix HI' (E20- 
(A)

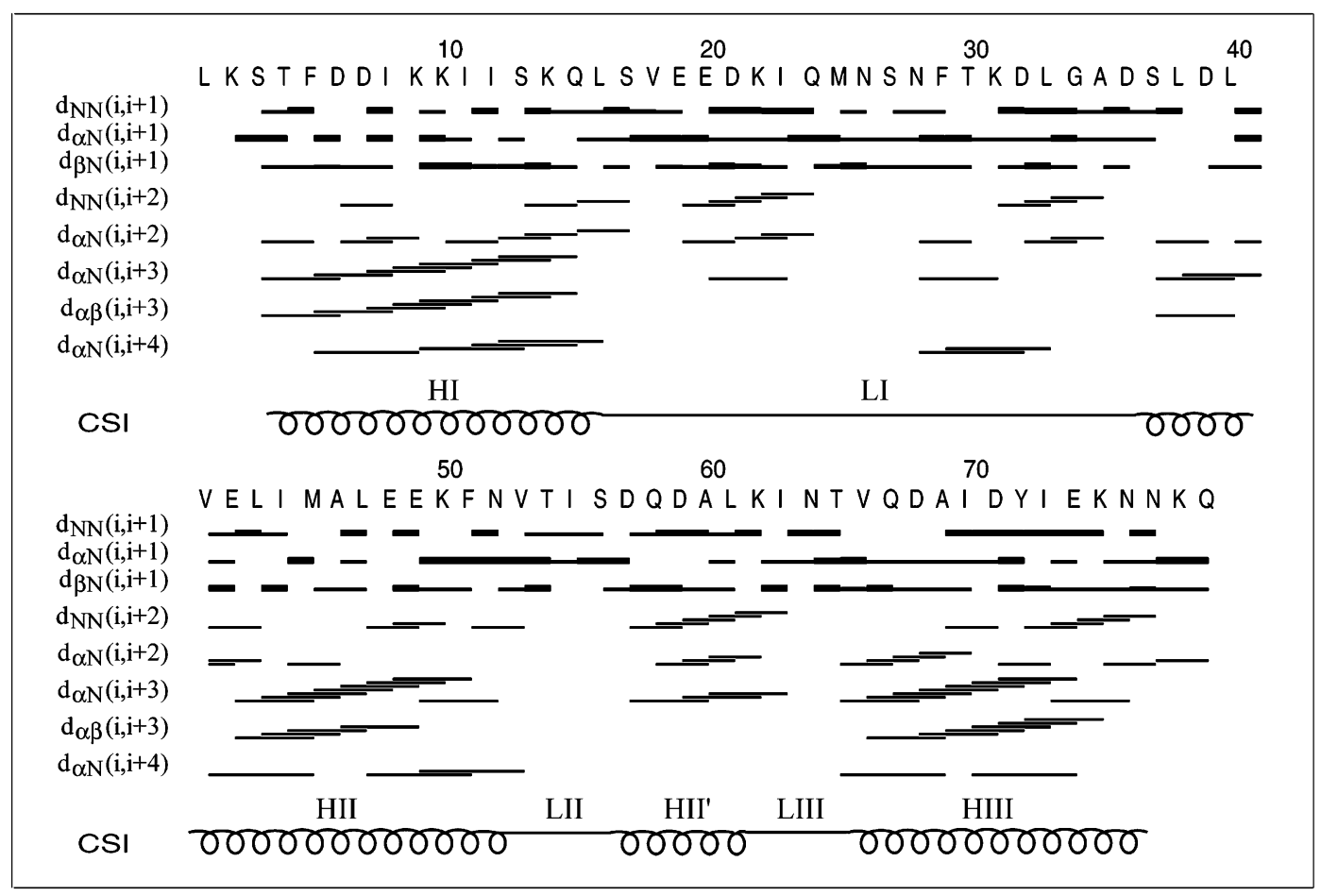

(B)

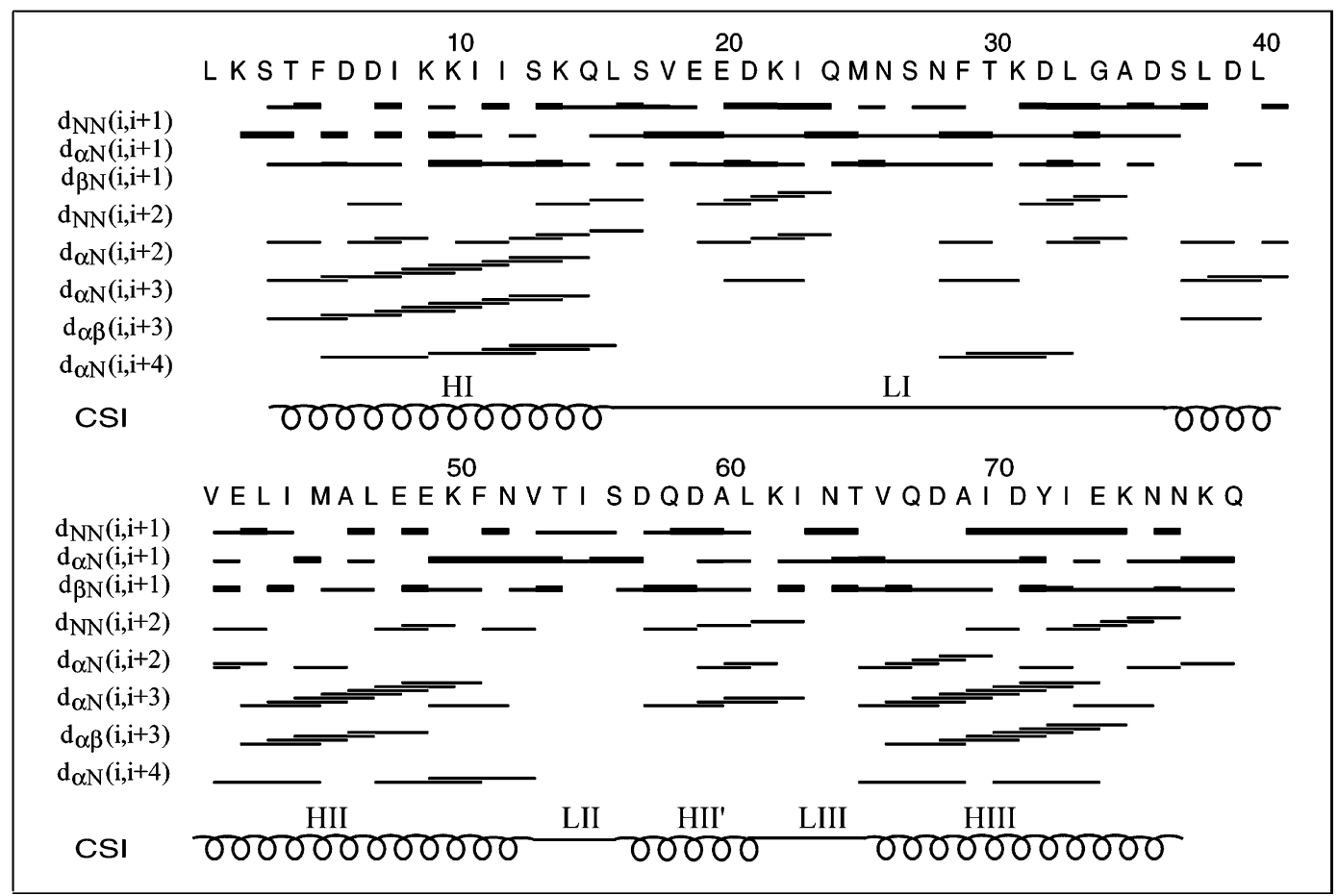

FIGURE 4: (A) Summary of sequential- and medium-range NOEs assigned in $3 \mathrm{D}{ }^{13} \mathrm{C}$ - and ${ }^{15} \mathrm{~N}$-edited NOESY-HSQC spectra for (A) the major conformation of holo-PfACP and (B) the minor conformation of holo-PfACP. The thickness of each bar is in proportion to the NOE intensity. Also shown in the figures is the secondary structure assignment for both the major and minor conformations of holo-PfACP as predicted by CSI.

I23). The presence of helix $\mathrm{HI}^{\prime}$ was not predicted on the basis of CSI and essentially arises because of sequence specific medium-range NOEs characteristic of helical structure. The presence of such an additional helix has been reported in structural studies of frenolicin $\mathrm{ACP}(17), E$. coli $\operatorname{ACP}(19,20)$, and apo-D-alanyl carrier protein (50). The mean structure of the major conformation of holo-PfACP consists of one additional helix HII' (D57-A60). The helices are connected by well-structured loops. The mean structure of the minor conformation lacks helix HII' (13 out of 20 structures lack helix $\mathrm{HII}^{\prime}$ ) despite a CSI-based prediction of a helix in this region. A total of 57 and $52 \%$ of the residues are part of helical segments in the major and minor conformers of holo-PfACP, respectively.

Major helices HI, HII, and HIII of holo-PfACP follow an up-down-down topological arrangement in the overall ar- 
(A)

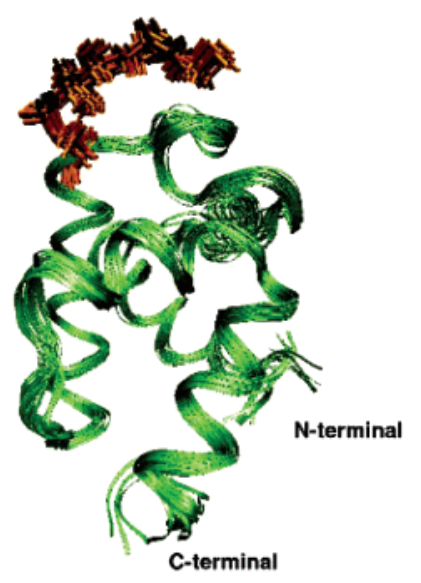

(C)

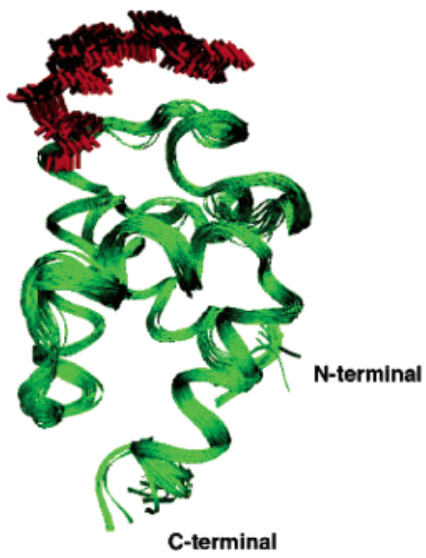

(B)

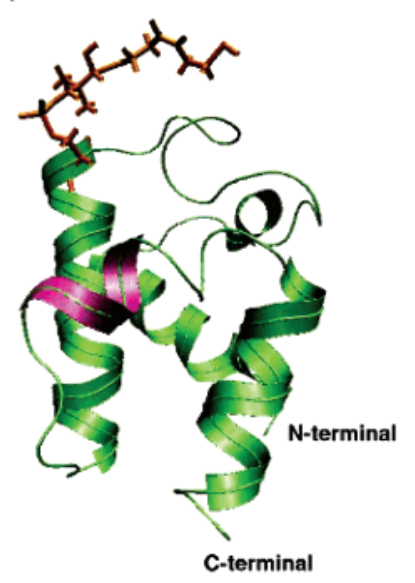

(D)

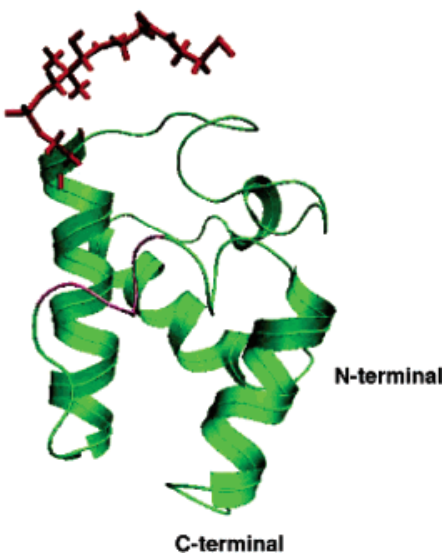

FIGURE 5: Solution NMR structures of holo-PfACP. (A) Backbone (superimposed on N, $\mathrm{C}^{\alpha}$, and $\mathrm{C}^{\prime}$ atoms) representation of an ensemble of 20 low-energy conformers of the major conformation. (B) Ribbon representation of a representative conformer of the major conformation. The 4'-phosphopantetheinylated serine in (A) and (B) is shown in orange. (C) Backbone (superimposed on $\mathrm{N}, \mathrm{C}^{\alpha}$, and $\mathrm{C}^{\prime}$ atoms) representation of an ensemble of 20 low-energy conformers of the minor conformation. (D) Ribbon representation of a representative conformer of the minor conformation. The 4'-phosphopantetheinylated serine in (C) and (D) is shown in red. Helix HII' in (B) and the loop region corresponding to helix HII' in (D) are purple.

chitecture. This topology is well conserved among the ACPs. Table 2 summarizes the structural statistics obtained for an ensemble of 20 solution structures of major and minor forms of holo-PfACP. The atomic rmsd for the ensemble of major and minor conformers with respect to their mean coordinate position when superposed on the backbone and all heavy atoms of residues $4-76$, are $0.48 \pm 0.09,0.92 \pm 0.10$, and $0.49 \pm 0.08,0.97 \pm 0.11 \AA$, respectively. Ramachandran statistics (51) show that 98.7 and $99.2 \%$ of the backbone dihedral angles lie in the allowed regions for major and minor conformations, respectively. Only 2 out of 79 residues, viz., $\mathrm{S} 17$ and S56, lie in the disallowed region. These residues lie in loops LI and LII, respectively. The low rmsd values and the high percentage of residues occupying the allowed regions in the Ramachandran map are an indication of high precision and the good stereochemical quality of calculated holo-PfACP structures.

The loop regions in both of the structures of holo-PfACP are as well defined as the helices. This is primarily due to the fact that 76 and 79 out of 146 long-range NOEs are between residues that are located in loops and helices for major and minor structures, respectively. The longest loop, LI, is packed between the three major helices, and its position is defined by 36 long-range NOEs to these helices for both of the structures. Similarly, loop LII is defined by 40 and
43 long-range NOEs to the helical segments for major and minor structures, respectively.

The native 3D fold of both of the conformations of holoPfACP is packed and stabilized primarily by hydrophobic interactions among and between such residues in helices and loops, with helix HII being most hydrophobic among helices in PfACP. This tight packing also explains the high order exhibited by the loops in this protein.

The rmsd when the mean structures of major and minor conformers of holo-PfACP are superimposed on their backbone atoms of helices HI, HII, and HIII regions is 0.341 $\AA$. The largest difference in the NMR-derived major and minor conformers is in the orientation of the $4^{\prime}$-PP prosthetic group. There also appears to be a significant deviation in loop LI, inspite of the lack of heterogeneity. The effect of this conformational heterogeneity of the prosthetic group on the structures of holo-PfACP will be discussed below.

Backbone Dynamics of Holo-PfACP. $T_{1}$ and $T_{2}$ relaxation rates and $\left\{{ }^{1} \mathrm{H}\right\}-{ }^{15} \mathrm{~N}$ heteronuclear NOE parameters have been determined for the protein backbone and the 4'-PP amide nitrogen atoms for both equilibrium forms of holo-PfACP. The measured relaxation parameters and order parameters $\left(S^{2}\right)$ of both forms as a function of amino acid sequence are shown in Figure 6. In general, the order parameter is a reflection of the rigidity of an internuclear $\mathrm{N}-\mathrm{H}$ bond vector 
Table 2: Structural Statistics of the Major and Minor Conformations of Holo-PfACP ${ }^{a}$

\begin{tabular}{|c|c|c|}
\hline & major & minor \\
\hline \multicolumn{3}{|c|}{ Distance Restraints (NOEs) } \\
\hline total & 1226 & 1218 \\
\hline intraresidue $(|\mathrm{i}-\mathrm{j}|)=0$ & 407 & 406 \\
\hline sequential $(|\mathrm{i}-\mathrm{j}|)=1$ & 329 & 322 \\
\hline medium range $(|\mathrm{i}-\mathrm{j}|)=2,3,4$ & 334 & 332 \\
\hline long range $(|i-j|)>4$ & 146 & 146 \\
\hline intra 4'-PP NOEs & 5 & 6 \\
\hline inter $4^{\prime}$-PP and protein NOEs & 5 & 6 \\
\hline average restraints per residue & 16.2 & 16.1 \\
\hline hydrogen bonds & 26 & 26 \\
\hline \multicolumn{3}{|l|}{ Dihedral angles } \\
\hline$\phi$ & 55 & 55 \\
\hline$\psi$ & 55 & 55 \\
\hline \multicolumn{3}{|c|}{ Structural Quality of Holo-PfACP } \\
\hline 1. Ramachandran map (\%) of residue $1-79$ & 67.9 & 68.6 \\
\hline most favored regions & 27.8 & 26.8 \\
\hline additionaly allowed regions & 3.0 & 3.8 \\
\hline $\begin{array}{l}\text { generously allowed regions } \\
\text { disallowed regions }\end{array}$ & 1.3 & 0.8 \\
\hline \multicolumn{3}{|l|}{ 2. restraint violation $(\AA)$} \\
\hline upper distance $(>0.5 \AA)$ & 0 & 0 \\
\hline dihedral angle $\left(>5^{\circ}\right)$ & 0 & 0 \\
\hline \multicolumn{3}{|l|}{$\begin{array}{l}\text { 3. average RMSD from mean coordinates }(\AA) \\
\text { (A) all residues }{ }^{b}\end{array}$} \\
\hline backbone atoms $\left(\mathrm{N}, \mathrm{C}^{\alpha}, \mathrm{C}^{\prime}\right)$ & $0.48 \pm 0.09$ & $0.49 \pm 0.08$ \\
\hline all heavy atoms & $0.92 \pm 0.10$ & $0.97 \pm 0.11$ \\
\hline \multicolumn{3}{|l|}{ (B) regular secondary structure elements ${ }^{c}$} \\
\hline backbone atoms & $0.25 \pm 0.05$ & $0.25 \pm 0.06$ \\
\hline all heavy atoms & $0.74 \pm 0.07$ & $0.76 \pm 0.07$ \\
\hline \multicolumn{3}{|l|}{ (C) loop region, residue range $16-36^{d}$} \\
\hline backbone atoms & $0.55 \pm 0.14$ & $0.58 \pm 0.15$ \\
\hline all heavy atoms & $1.09 \pm 0.23$ & $1.18 \pm 0.26$ \\
\hline \multicolumn{3}{|l|}{ (D) loop region, residue range $52-65^{e}$} \\
\hline backbone atoms & $0.55 \pm 0.14$ & $0.45 \pm 0.15$ \\
\hline all heavy atoms & $1.09 \pm 0.23$ & $0.87 \pm 0.17$ \\
\hline \multicolumn{3}{|c|}{${ }^{a}$ Statistics for the final ensemble of 20 structures calculated for holo- } \\
\hline \multicolumn{3}{|c|}{$\begin{array}{l}\text { PfACP. }{ }^{b} \text { The rmsd values for residue range } 4-76 .{ }^{c} \text { The residues of } \\
\text { helices HI( }(4-15), \mathrm{HII}(37-51) \text {, and HIII }(66-75) .^{d} \text { Excluding the residues } \\
\text { of helix HI'. }{ }^{e} \text { Excluding the residues of helix HII' in the major } \\
\text { conformation. }\end{array}$} \\
\hline
\end{tabular}

with respect to the principal axis of the molecule. The average values of $T_{1}, T_{2}$, and heteronuclear NOE are $0.444 \pm 0.010 \mathrm{~s}, 0.131 \pm 0.003 \mathrm{~s}$, and $0.701 \pm 0.014$ for the major conformation and $0.441 \pm 0.010 \mathrm{~s}, 0.133 \pm 0.003$ $\mathrm{s}$, and $0.702 \pm 0.014$ for the minor conformation of the polypeptide backbone. The residues in the three major helices all exhibit a tight distribution of $T_{1}, T_{2}$, and heteronuclear NOE values as well as high-order parameters, indicative of well-structured regions. The relaxation parameters for residues in loops LI, LII, and LIII also show a narrow distribution, although the lower-order parameters indicate conformational flexibility.

The relaxation parameters determined for the $\mathrm{N}-\mathrm{H}$ bond vectors of both isomeric forms of the prosthetic group, $4^{\prime}$ $\mathrm{PP}$, are indicated at positions 80 and 81 in Figure 6. The ${ }^{15} \mathrm{~N}$-relaxation parameters for the prosthetic group amides of the major conformation are as follows. For $4^{\prime}-\mathrm{PP}^{\mathrm{c}}$ : $T_{1}=$ $0.828 \pm 0.038 \mathrm{~s}, T_{2}=0.515 \pm 0.005 \mathrm{~s}$, and heteronuclear $\mathrm{NOE}=-0.634 \pm 0.013$; for $4^{\prime}-\mathrm{PP}^{\mathrm{g}}: T_{1}=0.643 \pm 0.013 \mathrm{~s}$, $T_{2}=0.204 \pm 0.002 \mathrm{~s}$, and heteronuclear NOE $=-0.114 \pm$ 0.002 . The similar relaxation parameters for the minor conformation are as follows. For $4^{\prime}-\mathrm{PP}^{\mathrm{c}}$ : $0.805 \pm 0.026 \mathrm{~s}$, $0.690 \pm 0.009 \mathrm{~s}$, and $-0.519 \pm 0.010$; for $4^{\prime}-\mathrm{PP}^{\mathrm{g}}: T_{1}=$
$0.617 \pm 0.006 \mathrm{~s}$, and $T_{2}=0.427 \pm 0.003 \mathrm{~s}$. The amide protons of the $4^{\prime}$-PP moiety show longer $T_{1}$ and $T_{2}$ relaxtion times as well as negative heteronuclear NOEs and low-order parameters. This is somewhat surprising in that distinct protein to prosthetic group internuclear NOEs can be assigned for each isomer. This would suggest that the prosthetic group does take on a more rigid conformation than can be inferred from relaxation data alone. It would be important to take into consideration exchange contributions such as those computed from relaxation-dispersion studies $(52,53)$. Furthermore, the measurement of dipolar couplings would also yield an accurate estimate of the order parameter for the prosthetic group.

Comparison of Holo-PfACP with Bacterial and Mammalian ACPs. The sequence alignment of ACPs that participate in type I and type II FAS pathways is shown in Figure 7A. Also shown in the Figure is the sequence of frenolicin ACP from the type II PKS pathway.

Multiple sequence alignment data indicate that the ACPs are almost similar in length except in the case of $M$. tuberculosis ACP, which is unique because of an extended $C$-terminal. PfACP shows 51.9, 48.8, 22.6, and $20.9 \%$ sequence identity with type II FAS ACP's from E. coli, $B$. subtilis, M. tuberculosis, and type I FAS rat ACP, respectively. Additionally, it shows a $23.8 \%$ sequence identity with $S$. roseofulvus ACP from type II PKS. An analysis of alignment data shows that the residues in the sequence that are most highly conserved are those that are adjacent to and form helix II and loop II structural regions. Furthermore, this region contains the functionally important DSL motif, to which the $4^{\prime}-\mathrm{PP}$ is tethered, with the conserved serine residue.

The structures of the major and minor conformations of holo-PfACP are compared to those of other ACPs, when superimposed on the backbone atoms of major helical regions, which are conserved among all ACPs. Such a comparison of the major conformation of holo-PfACP from type II FAS E. coli butyryl-ACP, M. tuberculosis ACP, and type I FAS rat ACP is shown in Figure $7(B-D)$. The overall fold of the PfACP is the same as that of the other ACPs, with conservation of the three-helix bundle. The major form of PfACP shows an rmsd of $2.24 \AA$ each with E. coli butyrylACP and M. tuberculosis ACP and $3.19 \AA$ with rat FAS I $\mathrm{ACP}$, whereas the minor form of holo-PfACP on similar comparison shows a rmsd of $2.19,2.35$, and $3.18 \AA$ to the respective ACPs. In the case where major and minor forms of PfACP are superimposed on the structure of frenolicin ACP and B. subtilis ACP, the rmsd is 2.16 and $2.58 \AA$ for the major form and 2.29 and $2.57 \AA$ for the minor form.

There is a striking difference in the surface-charge distribution if we compare PfACP with other members of the ACP family (Figure 7A). The surface of PfACP is not as highly acidic as in the case of other type II ACPs. The overall surface charge on PfACP is of -6 units (10 Lys, 10 Asp, and $6 \mathrm{Glu}$ ) residues. Helix $\mathrm{HII}$ is the most negatively charged among the helices in PfACP. From currently available sequence data on type II FAS ACPs, $M$. tuberculosis ACP is the most negatively charged, whereas PfACP carries the least negative charge. In contrast, the rat ACP of type I FAS is still less acidic with a net charge of -3 . In general, the bacterial ACPs have higher negative surface potential than the eukaryotic counterparts. Inspite of these 

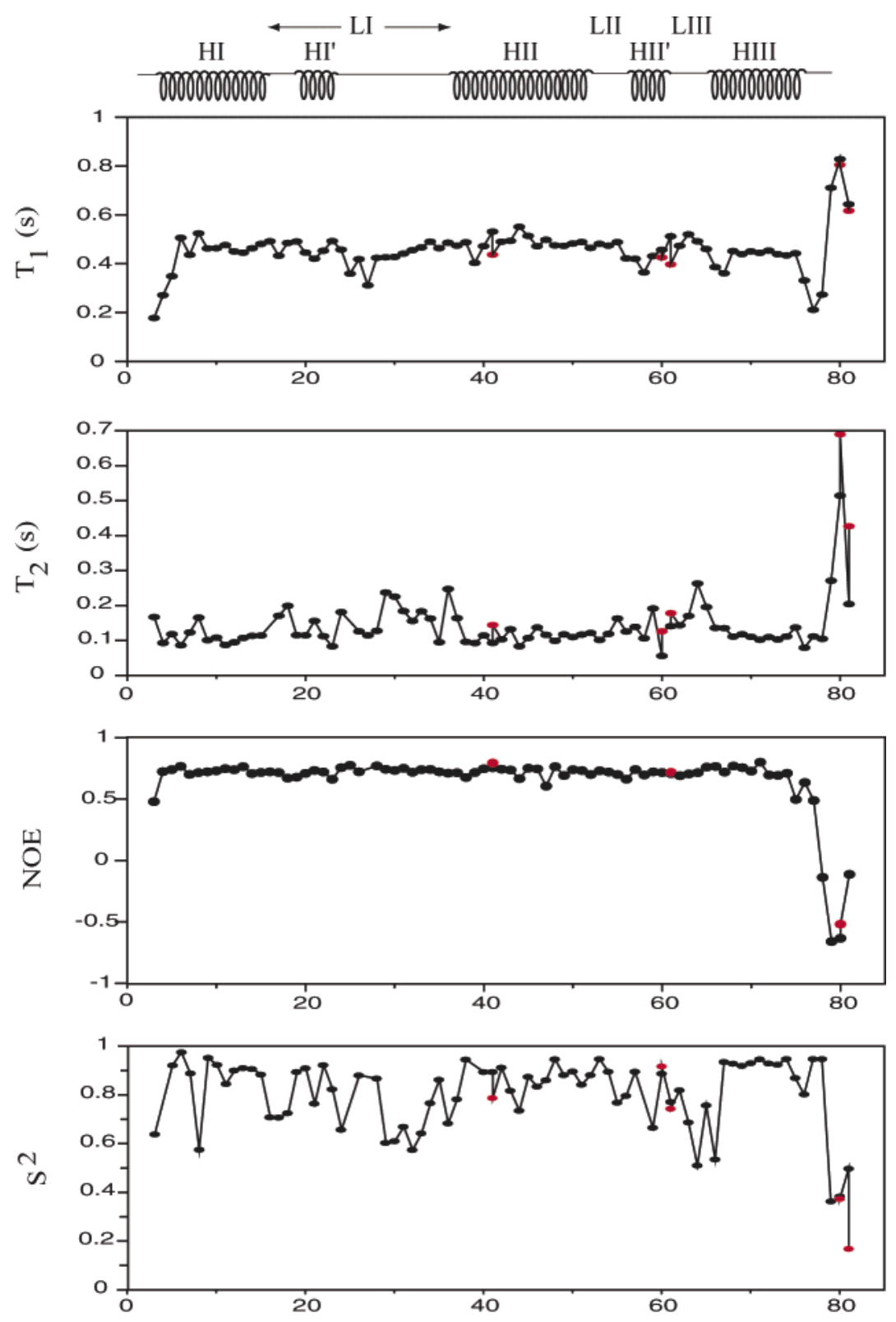

Residue Number

FIGURE 6: ${ }^{15} \mathrm{~N}$-relaxation data of holo-PfACP recorded at $300 \mathrm{~K}$. The values of measured relaxation times $T_{1}$ and $T_{2}$, steady-state heteronuclear NOEs, and calculated order parameters $\left(S^{2}\right)$ are plotted as a function of amino acid sequence. The relaxation data for $4^{\prime}-\mathrm{PP}^{\mathrm{c}}$ and $4^{\prime}-\mathrm{PP}^{\mathrm{g}}$ are shown in each plot as residue numbers 80 and 81, respectively. Similar values for the minor forms of conformationally exchanged residues are shown in red. Shown at the top of the plot is the secondary structure as a function of the sequence as deduced from the calculated tertiary structures for the equilibrium conformers. On the basis of the calculated structures, helix HII' is present only in the major conformer.

differences in overall charge, some common features are distinguishable in that the charge distribution in the vicinity of the prosthetic group appear to be the same. This coupled with the structural similarity among the various ACPs would explain the ability of ACP synthase (ACPS) from one organism to prime ACPs from other organisms (54-56). Analogously, it has been shown that apo-PfACP can be converted to the holo form by E. coli ACPS $(25,56,57)$.

A comparison of the structures of type II malaria ACP with type I mammalian ACP shows that there are pronounced differences in the overall structures as indicated by the small size of the helices and longer loop regions in rat ACP, the four helices being defined by $4,8,4$, and 5 residues. Figure 8 compares the structure of PfACP and rat apo-ACP. Electrostatic potential surfaces of PfACP and rat ACP are shown in Figure 8 (A and B). In the case of PfACP, the structure is stabilized by a dense network of hydrophobic interactions, which results in a tightly packed interior. The loop regions are relatively rigid, as evidenced by the higher than usual order parameters (cf. Figure 6). The rat ACP structure, however, shows far fewer hydrophobic interactions. Indeed, the rat ACP appears to possess a cavity in the interior as can be seen in Figure 8 (C and D).

\section{DISCUSSION}

The structures of acyl carrier proteins from several sources have been determined. The E. coli ACP in particular has been studied by both NMR (apo and holo forms) $(9-12)$ and X-ray crystallography (apo and acylated ACP) $(19,20)$. Solution NMR methods have shown that holo-ACP exists 
(A)

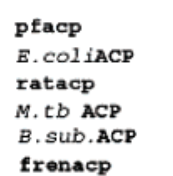

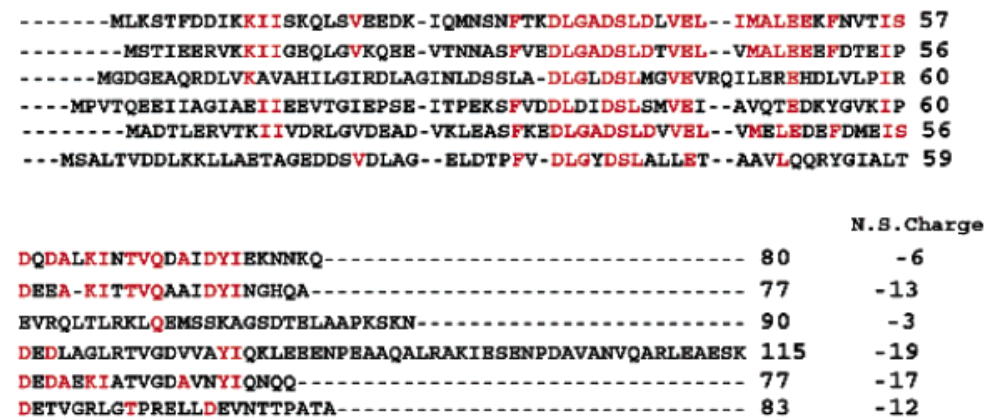

(B)

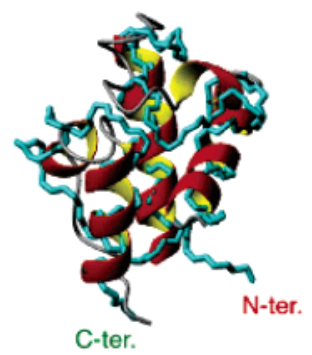

(C)

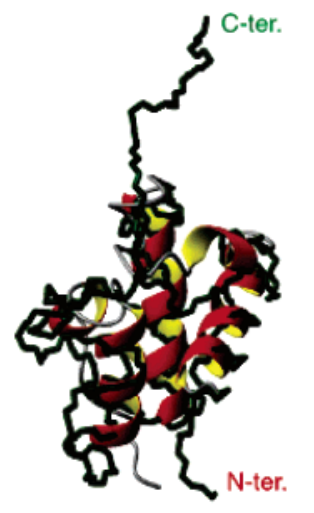

(D)

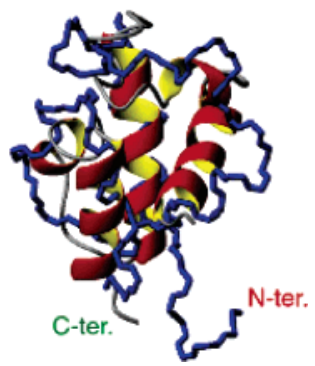

FIgURE 7: (A) Sequence alignment of PfACP with other ACPs (M. tb ACP $=$ M. tuberculosis ACP, B. sub. ACP $=B$. subtilis ACP, and fren $\mathrm{ACP}=$ frenolicin ACP). Manual adjustments were made following the initial alignment with ClustalW. Conserved residues are shown in red. The net surface charge (N.S.Charge) on ACPs were obtained by excluding terminal charges. (B-D) Overlays of the representative structure of the major conformation of PfACP on various ACPs with the superimposition of backbone atoms $\left(\mathrm{N}, \mathrm{C}^{\alpha}\right.$, and $\left.\mathrm{C}^{\prime}\right)$ on three major helices. (B) PfACP residues 4-15, 37-51, and 66-74 were superimposed on residues 4-15, 36-50, and 65-73 of E.coli butyryl ACP (pdb code 1L0I). (C) PfACP residues 4-15, 37-51, and 66-75 were superimposed on residues 8-19, 40-54, and 70-79 of M.tb ACP (pdb code 1KLP). (D) PfACP residues 4-7, 43-50, and 66-70 were superimposed on residues 9-12, 43-50, and 67-71 of rat FAS I ACP (pdb code 1N8L). The structure of PfACP is shown in ribbon representation, whereas those of E. coli butyryl ACP (cyan), M. tuberculosis ACP (green), and rat FAS I ACP (blue) are in backbone representation. See text for details.

as a dynamic equilibrium between two conformational states. Prestegard and co-workers have determined structures for both equilibrium forms of E. coli holo-ACP. Similar structural isomerism has also been observed in the case of frenolicin ACP (17) and spinach ACP (24), where NMR resonances for distinct equilibrium conformers could be identified and are present in a 10:1 ratio, indicating that these conformers are in equilibrium in the slow-exchange regime. For spinach and frenolicin ACPs, the time scale for chemical exchange is on the order of $50-160 \mathrm{~ms}$, based on the observation of exchange cross-peaks in NOESY spectra. In contrast, the conformational equilibrium exhibited by $E$. coli holo-ACP is on the fast-exchange regime on the NMR time scale in that a single set of correlation peaks was observed under all reported NMR experimental conditions. Furthermore, it has been shown that the 4'-PP group is present as an extended chain and does not exhibit any discernible interaction with the protein.

The holo-PfACP also exhibits conformational heterogeneity in solution. In this case, the major and minor forms are present in a ratio of 6.5:3.5, which is higher than that observed for other ACPs (17). More importantly, the prosthetic group has very clear interactions with the protein in both major and minor forms. Evidence for this comes from the NOESY data, where unique NOEs can be assigned to each form. The two conformers of holo-PfACP owe their origin to differences in interaction between the prosthetic group and the protein.
Differences in the orientation of the acylated prosthetic group has also been shown in the case of butyrylated $E$. coli ACP (20), which exists in open and closed conformations. In the closed conformation, the loop LII/helix HII' region (holo-PfACP notations) are packed against the three helix bundle and prevents the insertion of the lipophilic acyl group into the protein interior. In this conformation, the prosthetic group is thought to be flexible and in an extended conformation. In the open conformation, the loop LII/helix HII' region moves away from the three helix bundle, enabling the insertion of the acyl group into the hydrophobic pocket. Thus, the ACP must undergo a large conformational change in each successive cycle of chain elongation.

A comparison of similar regions, which in this case would be helix HII'/loop LIII, in the structures of the major and minor forms of holo-PfACP with E.coli butyryl-ACP (Figure 9) suggests that the major conformer more closely represents the closed conformation, whereas the minor one represents the open conformation. It also suggests that 4'-PP will have to undergo a conformational switch upon interaction with the first enzyme involved in the initiation of type II fatty acid bioynthesis, that is, FabD (malonyl CoA/ACP transacylase). It is possible that this conformational switch may play an important role in regulating fatty acid biosynthesis in the malarial parasite.

Changes in the orientation of the prosthetic group are also accompanied by changes in the tertiary structure. One important feature is the loss of helix HII' in the minor form 
(A)

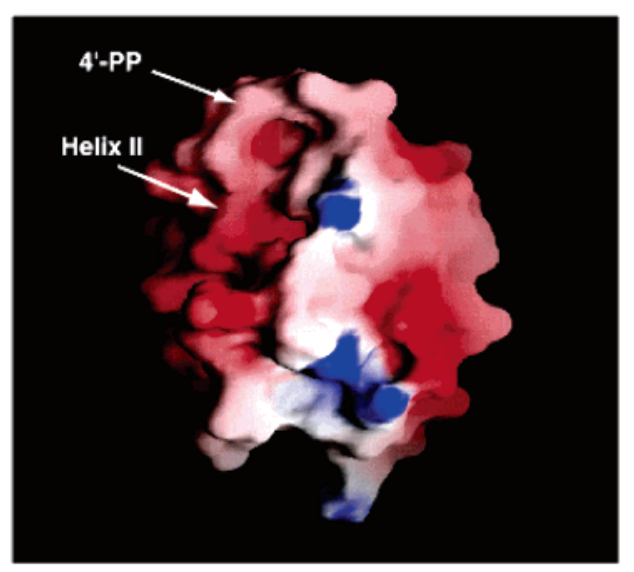

(C)

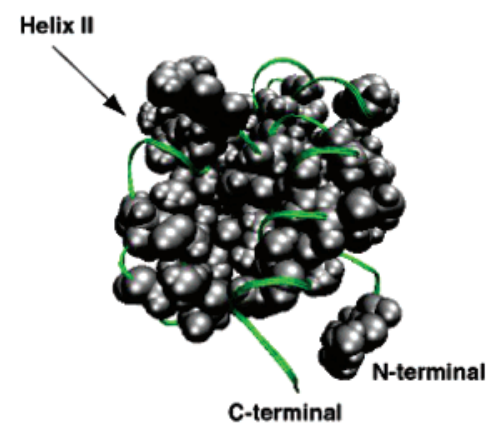

(D)
(B)
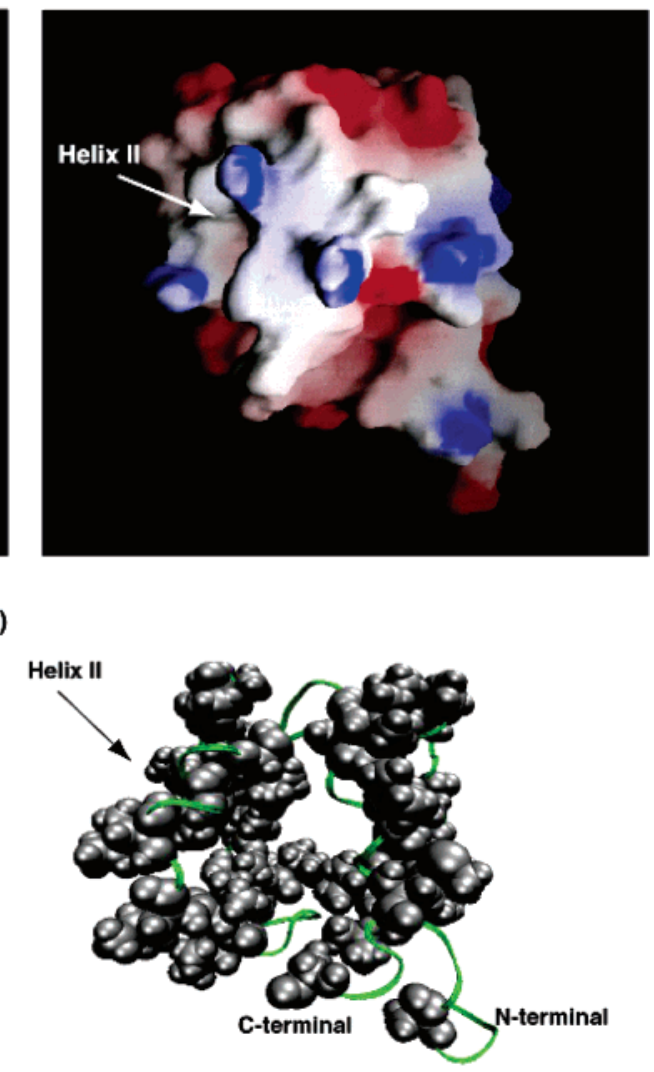

FIGURE 8: Electrostatic potential surface representation of (A) PfACP and (B) rat ACP and surface representation of the hydrophobic residues that form the interior hydrophobic cores of (C) PfACP and (D) rat ACP.

(A)

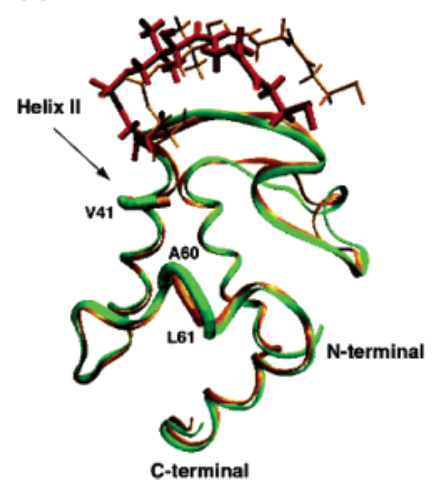

(B)

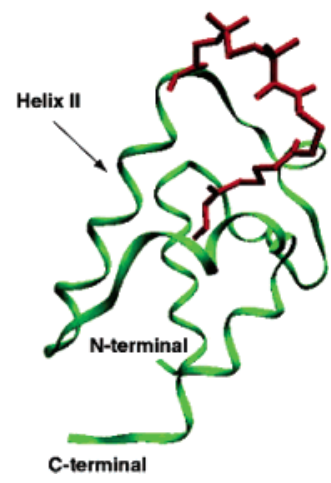

FIGURE 9: (A) Representative structures of the major and minor conformations of holo-PfACP superimposed on their backbone atoms $\left(\mathrm{N}, \mathrm{C}^{\alpha}\right.$, and $\left.\mathrm{C}^{\prime}\right)$ of helical regions are shown in orange and green, respectively. The 4'-phosphopantetheinylated serine is shown in orange and red for major and minor conformations, respectively. Residues that show conformational heterogeneity (V41, A60, and L61) are highlighted in tube representation. (B) E. coli butyryl$\mathrm{ACP}$ is shown in backbone representation and is green. The butyrylated prosthetic group along with serine-36 is shown in red.

and its conversion into a longer loop that connects helix HII and helix HIII. This is consistent with the open structure conformation observed in E. coli butyryl-ACP (Figure 9B). The additional flexibility in this region is necessary for the insertion of acyl chains into the hydrophobic pocket. The low rmsd of $0.341 \AA$ (Figure 9A) between the two conformational structures of holo-PfACP is indicative of an otherwise close structural similarity. Earlier studies on holoACP have shown that the linked acyl chain significantly interacts with the protein. However, interactions between the

prosthetic group and protein have not been observed in other ACPs $(58,59,9,13,14,17)$. In this study, the prosthetic group has been found to interact with several residues in both the major and minor forms (cf. Figures 2 and 3). All of the residues that interact with $4^{\prime}$-PP are present in helix HII.

From the current database, the structures of mammalian apo-ACP from the rat and the malaria parasite holo-ACP are available representative structures of ACPs from the host and parasite, respectively, and could also serve as canonical models for the comparison of ACPs that participate in type I and type II FAS pathways. As shown above, the differences in structures of these two proteins can be qualitatively described in terms of overall structure, surface charge, and stabilization of the tertiary structure by hydrophobic interactions. In terms of overall structure, the rat ACP shows a substantially lower order than PfACP. It is possible that the rat apo-ACP is intrinsically flexible. The overall charge differences between rat ACP and PfACP are marginal (cf. Figure $8 \mathrm{~A}$ and $\mathrm{B}$ ). An analysis of the thermodynamic properties could yield insights into the differences in stability of the two proteins. A point to consider is that the rat holoACP may present structural features that could be quite different from that of the apo-protein. A detailed comparison between the host and parasite ACPs will become possible once the structure of a mammalian holo-ACP is reported. E. coli ACPS will modify the apo forms of several type II ACP homologues including the Streptomyces ACPs of the PKS system. Not surprisingly, apo-PfACP is converted in its entirety to the holo form by nonrecombinantly expressed E. coli ACPS, even under conditions of stringent growth such as those encountered by cultures grown in a minimal medium 
(25). Similar conversion levels have been obtained in in vitro experiments too. In contrast, approximately $5 \%$ of the rat apo-ACP is converted to holo form in conditions where the E. coli ACPS is coexpressed. Similar levels of rat holo-ACP are obtained when the gene is overexpressed in $S$. coelicolor (60). Significantly, the rat holo-ACP can participate in the type II PKS system, although at extremely low efficiency. The ability of $S$. coelicolor ACPS to convert apo-PfACP to holo-PfACP is as yet unknown. In light of the above, there is a significant difference in specificity of $E$. coli ACPS for type II PfACP and type I rat ACP, respectively. It is possible that the differences in structure, between the Plasmodium and rat ACPs, shown here could be a major determinant of this specificity.

In conclusion, we have determined the solution structure of the holo-acyl carrier protein from the malaria parasite $P$. falciparum. The structure of this protein presents novel features that have not been observed in other ACPs. The differences in dynamical properties among ACPs leads to subtle differences in structure beyond the similar overall tertiary fold criterion in comparing the structures of homologous proteins from different species. Future studies on the interaction of holo-PfACP with $F a b D$ will reveal the role of conformational isomerism in this important proteinprotein interaction.

\section{ACKNOWLEDGMENT}

A.K.S. is a recipient of a Research Associateship from the Proteomics Initiative program of the Government of India. S.K.S. is a senior research fellow supported by CSIR, Government of India. S.P.S. acknowledges support from DBT and DST for the Biomolecular NMR facility at the Indian Institute of Science.

\section{SUPPORTING INFORMATION AVAILABLE}

$T_{1}$ and $T_{2}$ relaxation times, $\left\{{ }^{1} \mathrm{H}\right\}-{ }^{15} \mathrm{~N}$ heteronuclear NOEs, and order parameters $\left(S^{2}\right)$ along with their uncertainity values for both equilibrium forms of holo-PfACP. This material is available free of charge via the Internet at http://pubs.acs.org.

\section{REFERENCES}

1. World Health Organization. (2002) Reducing Risks, Promoting Healthy Life, The World Health Report 2002: Geneva, Switzerland.

2. Sibley, C. H., and Hunt, S. Y. (2003) Drug resistance in parasites: can we stay ahead of the evolutionary curve? Trends Parasitol. 19, 532-537.

3. White, N. J. (2004) Antimalarial drug resistance, J. Clin. Invest. $113,1084-1092$.

4. Surolia, N., and Surolia, A. (2001) Triclosan offers protection against blood stages of malaria by inhibiting enoyl-ACP reductase of Plasmodium falciparum, Nat. Med. 7, 167-173.

5. Waller, R. F., Ralph, S. A., Reed, M. B., Su, V., Douglas, J. D., Minnikin, D. E., Cowman, A. F., Besra, G. S., and McFadden, G. I. (2003) A type II pathway for fatty acid biosynthesis presents drug targets in Plasmodium falciparum, Antimicrob. Agents Chemother. 47, 297-301.

6. White, S. W., Zheng, J., Zhang, Y.-M., and Rock, C. O. (2005) The structural biology of type II fatty acid biosynthesis, Annu. Rev. Biochem. 74, 791-831.

7. Surolia, A., Ramya, T. N. C., Ramya, V., and Surolia, N. (2004) 'FAS't inhibition of malaria, Biochem. J. 383, 401-412.

8. Campbell, J. W., and Cronan, J. E., Jr. (2001) Bacterial fatty acid biosynthesis: targets for antibacterial drug discovery, Annи. Rev. Microbiol. 55, 305-332.
9. Holak, T. A., Kearsley, S. K., Kim, Y., and Prestegard, J. H. (1988) Three-dimensional structure of acyl carrier protein determined by NMR pseudoenergy and distance geometry calculations, Biochemistry 27, 6135-6142.

10. Holak, T. A., Nilges, M., Prestegard, J. H., Gronenborn, A. M., and Clore, G. M. (1988) Three-dimensional structure of acyl carrier protein in solution determined by nuclear magnetic resonance and the combined use of dynamical simulated annealing and distance geometry, Eur. J. Biochem. 175 (1), 9-15.

11. Holak, T. A., Nilges, M., and Oschkinat, H. (1989) Improved strategies for the determination of protein structures from NMR data: the solution structure of acyl carrier protein, FEBS Lett. 242, 218-224.

12. Kim, Y., and Prestegard, J. H. (1990) Refinement of the NMR structures for acyl carrier protein with scalar coupling data, Proteins (3rd Ed.) 8, 377-385.

13. Xu, G.-Y., Tam, A., Lin, L., Hixon, J., Fritz, C. C., and Powers, R. (2001) Solution structure of $B$. subtilis acyl carrier protein, Structure 9, 277-287.

14. Wong, H. C., Liu, G., Zhang, Y.-M., Rock, C. O., and Zheng, J. (2002) The solution structure of acyl carrier protein from Mycobacterium tuberculosis, J. Biol. Chem. 277, 15874-15880.

15. Reed, M. A. C., Schweizer, M., Szafranska, A. E., Arthur, C., Nicholson, T. P., Cox, J. R., Crosby, J., Crump, M. P., and Simpson, T. J. (2003) The type I rat fatty acid synthase ACP shows structural homology and analogous biochemical properties to type II ACPs, Org. Biomol. Chem. 1, 463-471.

16. Crump, M. P., Crosby, J., Dempsey, C. E., Parkinson, J. A., Murray, M., Hopwood, D. A., and Simpson, T. J. (1997) Solution structure of the actinorhodin polyketide synthase acyl carrier protein from Streptomyces coelicolor A3(2), Biochemistry 36, 6000-6008.

17. Li, Q., Khosla, C., Puglisi, J. D., and Liu, C. W. (2003) Solution structure and backbone dynamics of the holo form of the frenolicin acyl carrier protein, Biochemistry 42, 4648-4657.

18. Findlow, S. C., Winsor, C., Simpson, T. J., Crosby, J., and Crump, M. P. (2003) Solution structure and dynamics of oxytetracycline polyketide synthase acyl carrier protein from Streptomyces rimosus, Biochemistry 42, 8423-8433.

19. Qiu, X., and Janson, C. A. (2004) Structure of apo acyl carrier protein and a proposal to engineer protein crystallization through metal ions, Acta Crystallogr., Sect. D 60, 1545-1554.

20. Roujeinikova, A., Baldock, C., Simon, W. J., Gilroy, J., Baker, P. J., Stuitje, A. R., Rice, D. W., Slabas, A. R., and Rafferty, J. B. (2002) X-ray crystallographic studies on butyryl-ACP reveal flexibility of the structure around a putative acyl chain binding site, Structure 10, 825-835.

21. Parris, K. D., Lin, L., Tam, A., Mathew, R., Hixon, J., Stahl, M., Fritz, C. C., Seehra, J., and Somers, W. S. (2000) Crystal structures of substrate binding to Bacillus subtilis holo-(acyl carrier protein) synthase reveal a novel trimeric arrangement of molecules resulting in three active sites, Struct. Folding Des. 8, 883-895.

22. Zhang, Y. M., Marrakchi, H., White, S. W., and Rock, C. O. (2003) The application of computational methods to explore the diversity and structure of bacterial fatty acid synthase, J. Lipid Res. 44, $1-10$

23. Kim, Y., and Prestegard, J. H. (1989) A dynamic model for the structure of acyl carrier protein in solution, Biochemistry 28, 8792-8797.

24. Kim, Y., and Prestegard, J. H. (1990) Demonstration of a conformational equilibrium in acyl carrier protein from spinach using rotating frame nuclear magnetic resonance spectroscopy, J. Am. Chem. Soc. 112, 3707-3709.

25. Sharma, S. K., Modak, R., Sharma, S., Sharma, A. K., Sarma, S. P., Surolia, A., and Surolia, N. (2005) A novel approach for overexpression, characterization, and isotopic enrichment of a homogeneous species of acyl carrier protein from Plasmodium falciparum, Biochem. Biophys. Res. Commun. 330, 1019-1026.

26. McIntosh, L. P., and Dahlquist, F. W. (1990) Biosynthetic incorporation of ${ }^{15} \mathrm{~N}$ and ${ }^{13} \mathrm{C}$ for assignment and interpretation of nuclear magnetic resonance spectra of proteins, $Q$. Rev. Biophys. $23,1-38$.

27. Marion, D., Ikura, M., Tschudin, R., and Bax, A. (1989) Rapid recording of 2D NMR spectra without phase cycling. Application to the study of hydrogen exchange in proteins, J. Magn. Reson. $85,393-399$. 
28. Delaglio, F., Grzesiek, S., Vuister, G. W., Zhu, G., Pfeifer, J., and Bax, A. (1995) NMRPipe: a multidimensional spectral processing system based on UNIX pipes, J. Biomol. NMR 6, 277293.

29. Kraulis, P. J. (1989) ANSIG: a program for the assignment of protein $1 \mathrm{H}$ 2D NMR spectra by interactive graphics, J. Magn. Reson. 84, 627-633.

30. Kraulis, P. J., Domaille, P. J., Campbell-Burk, S. L., Van Aken, T., and Laue, E. D. (1994) Solution structure and dynamics of ras p21.GDP determined by heteronuclear three- and fourdimensional NMR spectroscopy, Biochemistry 33 (12), 35153531.

31. Cavanagh, J., Fairbrother, W. J., Palmer, A. G., and Skelton, N. J. (1996) Protein NMR Spectroscopy: Principles and Practice, Academic Press, Inc., San Diego, CA.

32. Bax, A., and Grzesiek, S. (1993) Methodological advances in protein NMR, Acc. Chem. Res. 26, 131-138.

33. Muhandiram, D. R., Farrow, N. A., Xu, G. Y., Smallcombe, S. H., and Kay, L. E. (1993) A gradient ${ }^{13} \mathrm{C}$ NOESY-HSQC experiment for recording NOESY spectra of ${ }^{13} \mathrm{C}$-labeled proteins dissolved in H2O, J. Magn. Reson., Ser. B 102, 317-321.

34. Vuister, G. W., and Bax, A. (1993) Quantitative J correlation: a new approach for measuring homonuclear three-bond $\mathrm{J}\left(\mathrm{H}^{\mathrm{N}} \mathrm{H}^{\alpha}\right)$ coupling constants in ${ }^{15} \mathrm{~N}$-enriched proteins, J. Am. Chem. Soc. $115,7772-7777$

35. Friebolin, H. (2005) Basic One- and Two-Dimensional NMR Spectroscopy, Wiley-VCH, Weinheim, Germany.

36. Farrow N. A., Muhandiram, R., Singer, A. U., Pascal, S. M., Kay, C. M., Gish, G., Shoelson, S. E., Pawson, T., Forman-Kay, J. D., and Kay, L. E. (1994) Backbone dynamics of a free and phosphopeptide-complexed Src homology 2 domain studied by 15N NMR relaxation, Biochemistry 33, 5984-6003.

37. Lipari, G., and Szabo, A. (1982a) Model-free approach to the interpretation of nuclear magnetic resonance relaxation in macromolecules. 1. Theory and range of validity, J. Am. Chem. Soc. $104,4546-4559$

38. Lipari, G., and Szabo, A. (1982b) Model-free approach to the interpretation of nuclear magnetic resonance relaxation in macromolecules. 2. Analysis of experimental results, J. Am. Chem. Soc. 104, 4559-4570.

39. Mandel, A. M., Akke, M., and Palmer, A. G. (1995) Backbone dynamics of Escherichia coli Ribonuclease HI: correlations with structure and function in an active enzyme, J. Mol. Biol. 246, 144- 163 .

40. Palmer, A. G., Rance, M., and Wright, P. E. (1991) Intramolecular motions of a zinc finger DNA-binding domain from Xfin characterized by proton-detected natural abundance carbon-13 heteronuclear NMR spectroscopy, J. Am. Chem. Soc. 113, 43714380.

41. Güntert, P., Mumenthaler, C., and Wüthrich, K. (1997) Torsion angle dynamics for NMR structure calculation with the new program DYANA, J. Mol. Biol. 273, 283-298.

42. Cornilescu, G., Delaglio, F., and Bax, A. (1999) Protein backbone angle restraints from searching a database for chemical shift and sequence homology, J. Biomol. NMR 13, 289-302.

43. Laskowski, R. A., Rullmann, J. A. C., MacArthur, M. W., Kaptein, R., and Thornton, J. M. (1996) AQUA and PROCHECK-NMR: programs for checking the quality of protein structures solved by NMR, J. Biomol. NMR 8, 477-486.

44. Koradi, R. B., Billeter, M., and Wüthrich, K. (1996) MOLMOL: a program for display and analysis of macromolecular structures, J. Mol. Graphics 14, 51-5, 29-32.
45. Humphrey, W., Dalke, A., and Schulten, K. (1996) VMD: visual molecular dynamics, J. Mol. Graphics 14, 33-8, 27-8.

46. Nicholls, A., Sharp, K., and Honig, B. (1991) Protein folding and association: insights from the interfacial and thermodynamic properties of hydrocarbons, Proteins: Struct., Funct., Genet. 11, $281-296$.

47. Pearson, W. R., and Lipman, D. J. (1988) Improved tools for the biological sequence comparison, Proc. Natl. Acad. Sci. U.S.A. 85, 2444-2448.

48. Sharma, A. K., Sharma, S. K., Surolia, N., and Sarma, S. P. (2005) NMR assignment of the holo-ACP from malaria parasite Plasmodium falciparum, J. Biomol. NMR 32, 260.

49. Wishart, D. S., and Sykes, B. D. (1994) The 13C chemical-shift index: a simple method for the identification of protein secondary structure using 13C chemical-shift data, J. Biomol. NMR 4, 171180

50. Volkman, B. F., Zhang, Q., Debabov, D. V., Rivera, E., Kresheck, G. C., and Neuhaus, F. C. (2001) Biosynthesis of D-alanyllipoteichoic acid: the tertiary structure of apo-D-alanyl carrier protein, Biochemistry 40, 7964-7972.

51. Ramachandran, G. N., and Saisekharan, V. (1968) Conformation of polypeptides and proteins, Adv. Protein Chem. 23, 283-437.

52. Halle, B., and Denisov, V. P. (2001) Magnetic relaxation dispersion studies of biomolecular solutions, Methods Enzymol. 338, 178201

53. Akke, M., Liu, J., Cavanagh, J., Erickson, H. P., and Palmer, A. G., III. (1998) Pervasive conformational fluctuations on microsecond time scales in a fibronectin type III domain, Nat. Struct. Biol. 5, 55-59.

54. Lambalot, R. H., Gehring. A. M., Flugel, R. S., Zuber, P., LaCelle, M., Marahiel, M. A., Reid, R., Khosla, C., and Walsh, C. T. (1996) A new enzyme superfamily - the phosphopantetheinyl transferases, Chem. Biol. 3, 923-936.

55. Gehring, A. M., Lambalot, R. H., Vogel, K. W., Drueckhammer, D. G., and Walsh, C. T. (1997) Ability of Streptomyces supp. acyl carrier proteins and coenzyme A analogs to serve as substrates in vitro for E.coli holo-ACP synthase, Chem. Biol. 4, 17-24.

56. Prigge, S. T. He, X. Gerena, L., Waters, N. C., and Reynolds, K. A. (2003) The initiating steps of a type II fatty acid synthase in Plasmodium falciparum are catalyzed by pfACP, pfMCAT, and pfKASIII, Biochemistry 42, 1160-1169

57. Waters, N. C., Kopydlowski, K. M., Guszczynski, T., Wei, L. Sellers, P., Ferlan, J. T., Lee, P. J., Li, Z., Woodard, C, L. Shallom, S., Gardner, M. J., and Prigge, S. T. (2002) Functional characterization of the acyl carrier protein (PfACP) and betaketoacyl ACP synthase III (PfKASIII) from Plasmodium falciparum, Mol. Biochem. Parasitol. 123, 85-94.

58. Mayo, K. H., and Prestegard, J. H. (1985) Acyl carrier protein from Escherichia coli. Structural charaterization of short-chain acylated acyl carrier proteins by NMR, Biochemistry 24, 78347838.

59. Jones, P.-J., Cioffi, E. A., and Prestegard, J. H. (1987) $\left\{{ }^{19} \mathrm{~F}\right\}-{ }^{1} \mathrm{H}$ Heteronuclear nuclear overhauser effect studies of the acyl chainbinding site of acyl carrier protein, J. Biol. Chem. 262, 89638965.

60. Tropf, S., Revill, W. P., Bibb, M. J., Hopwood, D. A., and Schweizer, M. (1998) Heterologously expressed acyl carrier protein domain of rat fatty acid synthase functions in Escherichia coli fatty acid synthase and Streptomyces coelicolor polyketide synthase systems, Chem. Biol. 5, 135-146.

$\mathrm{BI060368U}$ 